\title{
Antioxidant and Anti-Inflammatory Effects of Genus Gynura: A Systematic Review
}

\author{
Jiah Ning Tan ${ }^{1}$, Shamin Mohd Saffian ${ }^{2}$, Fhataheya Buang ${ }^{1}$, Zakiah Jubri ${ }^{3}$, Ibrahim Jantan ${ }^{4}$ \\ Khairana Husain ${ }^{1}$ and Norsyahida Mohd Fauzi ${ }^{1 *}$ \\ ${ }^{1}$ Centre for Drug and Herbal Development, Faculty of Pharmacy, Universiti Kebangsaan Malaysia, Kuala Lumpur, Malaysia, \\ ${ }^{2}$ Centre for Quality Management of Medicines, Faculty of Pharmacy, Universiti Kebangsaan Malaysia, Kuala Lumpur, Malaysia, \\ ${ }^{3}$ Department of Biochemistry, Faculty of Medicine, Universiti Kebangsaan Malaysia, Kuala Lumpur, Malaysia, ${ }^{4}$ Institute of \\ Systems Biology, Universiti Kebangsaan Malaysia, Bangi, Malaysia
}

Background: Gynura species have been used traditionally to treat various ailments, such as fever, pain, and to control blood glucose level. This systematic review critically discusses studies regarding Gynura species that exhibited antioxidant and anti-inflammatory effects, thus providing perspectives and instructions for future research of the plants as a potential source of new dietary supplements or medicinal agents.

\section{OPEN ACCESS}

Edited by: Marco Leonti,

University of Cagliari, Italy

Reviewed by:

Sayeed Ahmad,

Jamia Hamdard University, India

Md. Areeful Haque,

International Islamic University

Chittagong, Bangladesh

*Correspondence:

Norsyahida Mohd Fauzi drnorsyahida@ukm.edu.my

Specialty section:

This article was submitted to

Ethnopharmacology,

a section of the journal

Frontiers in Pharmacology

Received: 14 October 2019

Accepted: 13 October 2020

Published: 27 November 2020

Citation:

Tan JN, Mohd Saffian S, Buang F,

Jubri Z, Jantan I, Husain K and Mohd Fauzi N (2020) Antioxidant and Anti-Inflammatory Effects of Genus Gynura: A Systematic Review. Front. Pharmacol. 11:504624. doi: 10.3389/fphar.2020.504624
Methods: A literature search from internet databases of PubMed, Scopus, Science Direct, e-theses Online Service, and ProQuest was carried out using a combination of keywords such as "Gynura," "antioxidant," "anti-inflammatory," or other related words. Research articles were included in this study if they were experimental (in vitro and in vivo) or clinical studies on the antioxidant or anti-inflammatory effects of Gynura species and if they were articles published in English.

\footnotetext{
Abbreviations: $\mathrm{ADI}$, acceptable daily intake; $\mathrm{AOM}$, azoxymethane; $\mathrm{BW}$, body weight; $\mathrm{CAT}$, catalase; $\mathrm{CCl}_{4}$, carbon tetrachloride; COX-2, cyclooxygenase-2; CRP, c-reactive protein; DCFH-DA, 2' $7^{\prime}$-dichlorofluorescein diacetate; DMSO, dimethyl sulfoxide; EAE, enzyme-assisted extraction; EIA, enzyme immunoassay; ELISA, enzyme-linked immunosorbent assay; EMSA, electrophoretic mobility shift assay; ESI-MS, electrospray ionization-mass spectrometry; ESI-TOF-MS, electrospray ionization time-of-flight mass spectrometry; EThOS, e-theses online service; FRSA, free radical scavenging activity; GC-MS, gas chromatography-mass spectrometer; GPT, glutame pyruvate transaminase; GR, glutathione reductase; GSH, glutathione; GSHPx, glutathione peroxidase; GSK3 $\beta$, glycogen synthase kinase three beta; GSSG, glutathione disulfide; GST, glutathione-Stransferase; $\mathrm{H}$, human; $\mathrm{H}_{2} \mathrm{O}_{2}$, hydrogen peroxide; HDFs, human dermal fibroblasts; HPLC, high-performance liquid chromatography; HP-TLC, high-performance thin-layer chromatography; HSOS, sinusoidal obstruction syndrome; huvec, human umbilical vein endothelial cell; HVOD, hepatic veno-occlusive disease; i. p., intraperitoneal; $\mathrm{IC}_{50}$, inhibitory concentration $50 \%$; IFN- $\gamma$, interferon-gamma; IL, interleukin; iNOS, inducible nitric oxide synthase; LC-MS/MS, liquid chromatography with tandem mass spectrometry; LDH, lactate dehydrogenase; LPS, lipopolysaccharide; $\mathrm{MDA}$, malondialdehyde; $\mathrm{MeOH}$, methanol; MMP-1, matrix metalloproteinase-1; mRNA, messenger ribonucleic acid; NF- $k B$, nuclear factor kappa B; NGF, nerve growth factor; NMR, nuclear magnetic resonance; NO, nitric oxide; Nrf2, nuclear factor erythroid 2-related factor 2; OHAT, office health assessment and translation for conducting a systematic review and evidence integration; PBMCs, peripheral blood mononuclear cells; $\mathrm{PGE}_{2}$, prostaglandin $\mathrm{E}_{2}$; p-IкBa, phospho-IкB alpha; PMA, phorbol 12-myristate 13-acetate; PMNs, polymorphonuclear cells; PPAR $\gamma$, peroxisome proliferator-activated receptor gamma; PRISMA, preferred reporting items for systematic reviews and meta-analyses; ROS, reactive oxygen species; RT-PCR, reverse transcription polymerase chain reaction; SIRT, sirtuin; SOD, superoxide dismutase; STZ, streptozotocin; TAS, total antioxidant status; TBARS, thiobarbituric acid reactive substances; TFC, total flavonoid content; TNF- $\alpha$, tumor necrosis factor alpha; TPC, total phenolic content; UEAE, ultrasonic-enzyme-assisted extraction; UHPLC-Q-TOF-MS/MS, ultra-high-performance liquid chromatography-quadrupole time-of-flight mass spectrometry; UV, ultraviolet; $\mathrm{v} / \mathrm{v}$, volume/volume; $\mathrm{v} / \mathrm{w}$, volume/weight; $\Delta \psi \mathrm{m}$, mitochondrial membrane potential.
} 
Results: Altogether, 27 studies on antioxidant and anti-inflammatory effects of Gynura species were selected. The antioxidant effects of Gynura species were manifested by inhibition of reactive oxygen species production and lipid peroxidation, modulation of glutathione-related parameters, and enzymatic antioxidant production or activities. The anti-inflammatory effects of Gynura species were through the modulation of inflammatory cytokine production, inhibition of prostaglandin $\mathrm{E}_{2}$ and nitric oxide production, cellular inflammatory-related parameters, and inflammation in animal models. The potential antiinflammatory signaling pathways modulated by Gynura species are glycogen synthase kinase-3, nuclear factor erythroid 2-related factor 2, PPAR $\gamma, \mathrm{MAPK}, \mathrm{NF}-\mathrm{kB}$, and PI3K/Akt. However, most reports on antioxidant and anti-inflammatory effects of the plants were on crude extracts, and the chemical constituents contributing to bioactivities were not clearly understood. There is a variation in quality of studies in terms of design, conduct, and interpretation, and in-depth studies on the underlying mechanisms involved in antioxidant and anti-inflammatory effects of the plants are in demand. Moreover, there is limited clinical study on antioxidant and anti-inflammatory effects of Gynura species.

Conclusion: This review highlighted antioxidant and anti-inflammatory effects of genus Gynura and supported their traditional uses to treat oxidative stress and inflammatoryrelated diseases. This review is expected to catalyze further studies on genus Gynura. However, extensive preclinical data need to be generated from toxicity and pharmacokinetic studies before clinical studies can be pursued for their development into clinical medicines to treat oxidative stress and inflammatory conditions.

Keywords: Gynura, medicinal plant, reactive oxygen species, antioxidant, anti-inflammatory

\section{INTRODUCTION}

Inflammation refers to a complex array of defensive immune responses (Arulselvan et al., 2016), and tissue damage is one of the consequences of an exaggerated or uncontrolled prolonged inflammatory process (Biswas, 2016). Inflammatory cells, including neutrophils and macrophages, generate free radicals at the inflammation site, where reactive oxygen species (ROS) (e.g., hydroxyl radicals, superoxide anion radicals, and hydrogen peroxide) act as both signaling molecules and inflammation mediators. Enhancement of pro-inflammatory gene expression can be achieved via the initiation of the intracellular signaling cascade by reactive species. The exaggerated generation of reactive species in pathological inflammatory conditions may induce localized oxidative stress and tissue injury, thus promoting progression of many inflammatory diseases (Mittal et al., 2014). Hence, inflammation and oxidative stress are highly interdependent pathophysiological events in various types of chronic diseases (Vaziri and Rodríguez-Iturbe, 2006; Biswas, 2016).

A total of 46 species are identified in the genus Gynura (The Plant List, 2020). They are distributed from tropical Africa to South and East Asia as well as Australia where the highest specific diversity is found in Southeast Asia (Vanijajiva and Kadereit, 2011). The fresh leaves of $G$. procumbens (Lour.) Merr. are traditionally consumed to control blood glucose level by Orang Asli in Kampung Bawong, Perak, West Malaysia (Samuel et al., 2010). G. procumbens is also traditionally used to treat kidney discomfort, inflammation, rheumatic fever, and viral ailments (Wiart, 2006). G. pseudochina (L.) DC. is traditionally used to treat fever and sore eye (Davies, 1980). In Chinese folk medicine, G. segetum (Lour.) Merr. is consumed as a decoction or is soaked in wine and orally taken to promote microcirculation or relieve pain (Chen et al., 2011). In Nepal, the juice of G. nepalensis DC. is applied on cuts and wounds as healing treatment (Manandhar, 2002).

Incorporation of a plant-based natural antioxidant in daily diet can prevent several human illnesses (Knekt et al., 1996) as the prevalence of many illness is inversely linked to the dietary consumption of antioxidant-rich foods (Sies, 1993). For any herb to be developed into a dietary supplement, it is essential that the evidence for the claimed effects of the herb is scientifically demonstrated. Ideally, the mechanisms of action should also be understood. The potential use of Gynura as a phytomedicine with antioxidant and anti-inflammatory properties has been well documented, but we were unable to identify specific review articles that focused on the antioxidant and antiinflammatory effects of the Gynura species. This review was undertaken to assess published experimental data that investigated the antioxidant and anti-inflammatory activities of the Gynura species to support the design of future studies. It also provided an evaluation of the quality of available information, summarized mechanisms of action data from animal and cell studies following the administration of the Gynura species, and identified research gaps in the literature.

\section{METHODS}

This systematic review was carried out according to the Preferred Reporting Items for Systematic Reviews and Meta-Analyses (PRISMA) guideline (Moher et al., 2010). The quality of 
references was determined by referring to the Office Health Assessment and Translation for Conducting a Systematic Review and Evidence Integration (OHAT) guideline (Rooney et al., 2014).

\section{Search Strategy}

The information was obtained through a comprehensive literature search using the electronic databases of PubMed, Scopus, and Science Direct from 2000 to March 2020 for journal articles and the databases of e-theses Online Service and ProQuest Dissertations and Theses Global for theses. The combination of keywords used in PubMed was as follows: [Gynura (Title/Abstract)] AND [antioxidant (Title/Abstract)] OR [oxidant (Title/Abstract)] OR [oxidative (Title/Abstract)] OR [oxidation (Title/Abstract)] OR [antiinflammatory (Title/Abstract)] OR [antiinflammation (Title/ Abstract)] OR [inflammation (Title/Abstract)] OR [inflammatory (Title/Abstract)] OR [inflame (Title/Abstract)]. The reference lists of all included papers were checked for other potentially relevant citations. Studies selection was restricted to articles in English because of language barrier, time efficiency, and high cost for translation. However, only four studies were excluded on the basis of not being published in English, which is unlikely to impact our findings. In order to achieve a comprehensive search of relevant studies, university dissertations, and theses were accessed in the selection process. However, the confidentiality of these tools had possibly veiled some important information and results, for example, negative findings.

\section{Inclusion and Exclusion Criteria}

Studies were included in this review if they were experimental studies (in vitro and in vivo) or clinical studies on the antioxidant or anti-inflammatory effects of Gynura and if they were articles published in English. The Gynura plant can be from any parts of the plant and in any form such as extracts, essential oils, fractions, or isolated compounds. Studies were excluded if they met at least one of the following criteria: 1) study models were not accepted as evidence for pharmacological effects (i.e., antioxidant experiments of FRAP, ABTS, DPPH, and Trolox equivalent antioxidant capacity assays), and 2) intervention was not focused on Gynura, although the antioxidant or anti-inflammatory effects were measured. Review articles and book chapters were excluded from this study, but the references were mined to search for further relevant studies.

\section{Data Extraction and Handling}

The following details were extracted from each selected study: 1) the species and part of the Gynura used; 2) methods used, including animal species or cell lines, study design, and treatment details; 3) outcome measures; and 4) findings on the antioxidant and anti-inflammatory effects of Gynura.

\section{Quality Assessment}

The reporting completeness of the material used by each selected study was assessed using the information of the Gynura species material, voucher specimens, report on quality control of extract and chemical analysis. The risk of bias in the included studies was assessed by two investigators based on the OHAT guideline for any potential bias such as selection bias, performance bias, attrition or exclusion bias, detection bias, selection reporting bias, and other sources of bias. However, some of the questions in the bias assessment were excluded as they were not applicable for use in the assessment of in vitro, in vivo, and human randomized control study designs.

\section{RESULTS AND DISCUSSION}

\section{Study Selection}

A total of 183 studies were found from the database search, and 11 additional articles were identified from other sources. After removing the duplicates, 125 articles were shortlisted, and after title-abstract screening, 57 articles were excluded because of the following reasons: The articles were published in languages other than English $(n=4)$, the articles are non-experimental journal articles $(n=10)$, and the articles reported on the effects of Gynura other than antioxidant and anti-inflammatory $(n=43)$. By full-text screening of the remaining 68 articles, a total of 41 articles were eliminated based on the following exclusion criteria: study models used are not accepted as evidence for pharmacological effects ( $\mathrm{n}=$ $25)$, study intervention does not focus on Gynura $(\mathrm{n}=1)$, and a combination of both exclusion criteria $(n=15)$. Thus, 27 articles from year 2002 to 2020 were selected in the final qualitative analysis of this systematic review. Three potential dissertations and theses were accessed through the two databases. Only one of the theses was selected, but the result presented in the theses has been published in one of the article selected in this review (Siriwatanametanon et al., 2010). Other two theses were excluded because they did not fulfill the inclusion criteria. A flowchart depicting the search process and study selection is presented in Figure 1.

\section{Quality Assessment}

All selected articles were assessed for their quality and risk of bias. Table 1 shows the quality assessment of studies on the antioxidant and anti-inflammatory effects of Gynura species. The reporting completeness of the material in all selected studies was assessed using information of the Gynura species material, voucher specimens, quality control, and chemical analysis. Naming inconsistency between studies was detected as full botanical taxonomic names were not stated in 13 selected studies (Iskander et al., 2002; Siriwatanametanon and Heinrich, 2011; Akowuah et al., 2012; Seow et al., 2014; Chao et al., 2015; Wong et al., 2015; Xu et al., 2015; Rerknimitr et al., 2016; Yin et al., 2017; Rahman et al., 2018; Pai et al., 2019; Yang et al., 2019; Chandradevan et al., 2020). All plant species need to be validated taxonomically, and the full names have to be clearly stated as the incomplete name of plant species can lead to confusion of readers and any recorded uses or properties attributed may, in fact, correlate to different species. Such erroneous publications on the use of plant names are a permanent source of confusion for future research, search engines, and databases (Rivera et al., 2014). An incomplete plant name in some contexts may be ambiguous or may even mislead readers. This error is possible to occur within genus Gynura. Gynura divaricata is no guarantee to solely indicate Gynura divaricata (L.) DC. as Gynura divaricata subsp. barbareifolia (Gagnep.) F.G.Davies 


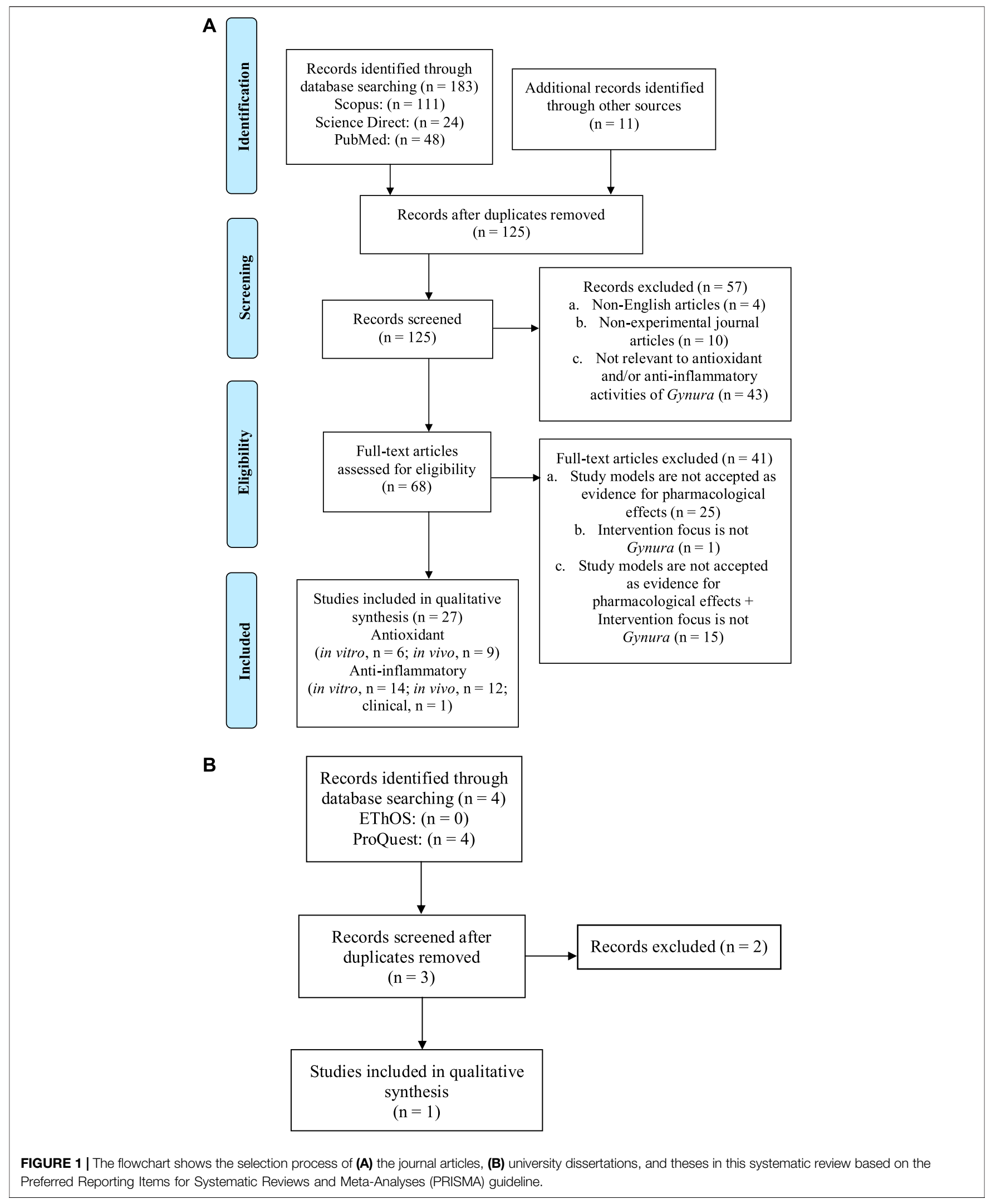


TABLE 1 | Quality assessment of studies on the antioxidant and anti-inflammatory effects of Gynura species.

\begin{tabular}{|c|c|c|c|c|c|}
\hline Study & $\begin{array}{l}\text { Species stated } \\
\text { in article }\end{array}$ & Plant source & $\begin{array}{l}\text { Authenticated } \\
\text { species }\end{array}$ & $\begin{array}{l}\text { Quality } \\
\text { control } \\
\text { reported? }\end{array}$ & $\begin{array}{l}\text { Chemical analysis } \\
\text { reported? }\end{array}$ \\
\hline Iskander et al. (2002) & Gynura procumbens & $\begin{array}{l}\text { Department of Pharmacognosy, Faculty of } \\
\text { Pharmacy, Mahidol University, Bangkok, Thailand }\end{array}$ & - & No & No \\
\hline Akowuah et al. (2012) & $\begin{array}{l}\text { Gynura procumbens (Merr., } \\
\text { compositae) }\end{array}$ & Penang Island, Malaysia & + & No & No \\
\hline Kim et al. (2011) & Gynura procumbens (Lour.) Merr & Specialty Natural Products Co. Ltd., Thailand & + & No & $\begin{array}{l}\text { Yes-HPLC, electrospray ionization time-of-flight } \\
\text { mass spectrometry }\end{array}$ \\
\hline Shwter et al. (2014) & Gynura procumbens (Lour.) Merr & Ethno Resources Sdn Bhd, Malaysia & + & No & No \\
\hline Wong et al. (2015) & Gynura procumbens & $\begin{array}{l}\text { Green House Facility, Faculty of Science and } \\
\text { Technology, Universiti Kebangsaan Malaysia (UKM) }\end{array}$ & + & No & No \\
\hline Huang et al. (2019) & Gynura procumbens (Lour.) Merr & Hainan Province, South China & + & No & Yes-GC-MS \\
\hline Liu M. et al. (2019) & Gynura procumbens (Lour.) Merr & Jing'an, Jiangxi Province, China & - & No & $\begin{array}{l}\text { Yes-ultra-high-performance liquid chromatography- } \\
\text { quadrupole time-of-flight mass spectrometry }\end{array}$ \\
\hline Nazri et al. (2019) & Gynura procumbens (Lour.) Merr & Semenyih, Selangor, Malaysia & + & No & Yes-LC-MS/MS \\
\hline Ning et al. (2019) & Gynura procumbens (Lour.) Merr & Brightmark Sdn. Bhd, Semenyih, Malaysia & + & No & No \\
\hline Liu Y. et al. (2019) & Gynura procumbens (Lour.) Merr & Shanxi Jintai Biol, China & _ & No & Yes-LC-MS/MS \\
\hline Chandradevan et al. (2020) & Gynura procumbens & $\begin{array}{l}\text { Net house located in the Malaysia Agricultural, } \\
\text { Research and development institute (MARDI) }\end{array}$ & + & No & Yes $-{ }^{1} \mathrm{H}$ NMR spectroscopy \\
\hline Wu et al. (2013) & Gynura bicolor (Roxb. and Willd.) DC. & Yuanshan Village, llan, Taiwan & + & No & Yes-HPLC \\
\hline Chao et al. (2015) & Gynura bicolor DC. & Farms in Puli Town, Nanton county, Taiwan & + & No & No \\
\hline Yin et al. (2017) & Gynura bicolor DC & Farms in Puli Town, Nanton county, Taiwan & + & No & No \\
\hline Pai et al. (2019) & Gynura bicolor & Farms, unknown location & - & No & No \\
\hline Yang et al. (2019) & Gynura bicolor DC. & Farms, unknown location & _- & No & No \\
\hline Siriwatanametanon et al. (2010) & $\begin{array}{l}\text { Gynura pseudochina (L.) DC. var. } \\
\text { hispida Thv }\end{array}$ & $\begin{array}{l}\text { Farmland in north-eastern part of Thailand, mainly in } \\
\text { Buriram province }\end{array}$ & + & No & No \\
\hline $\begin{array}{l}\text { Siriwatanametanon and Heinrich } \\
\text { (2011) }\end{array}$ & $\begin{array}{l}\text { Gynura pseudochina (L.) var. hispida } \\
\text { Thv }\end{array}$ & Not stated & - & No & $\begin{array}{l}\text { Yes-electrospray ionization-mass spectrometry, }{ }^{1} \mathrm{H} \\
\text { NMR, }{ }^{13} \mathrm{C} \text { NMR, 2D-NMR }\end{array}$ \\
\hline Rerknimitr et al. (2016) & $\begin{array}{l}\text { Gynura pseudochina DC. var. hispida } \\
\text { Thv }\end{array}$ & Not stated & - & No & No \\
\hline Sukadeetad et al. (2018) & Gynura pseudochina (L.) DC. & $\begin{array}{l}\text { Koeng Sub-district, Mueang District, Maha } \\
\text { Sarakham province, Thailand }\end{array}$ & + & No & Yes-HP-TLC, HPLC, LC-MS/MS \\
\hline Seow et al. (2014) & Gynura segetum & Jabatan Pertanian Relau, Penang, Malaysia & + & No & No \\
\hline Yuandani et al. (2017) & Gynura segetum (Lour.) Merr & Yogyakarta, West Java, Indonesia & + & No & Yes-HPLC \\
\hline Yu et al. (2016) & Gynura nepalensis DC. & Suburb of Shanghai, China & + & No & Yes-HPLC \\
\hline Rahman et al. (2018) & Gynura nepalensis & Kendua under Netrokona district of Bangladesh & + & No & Yes-method not stated \\
\hline Xu et al. (2015) & Gynura divaricata $L$ & $\begin{array}{l}\text { Silk Biotechnology Laboratory, Soochow University, } \\
\text { Suzhou, China }\end{array}$ & - & No & No \\
\hline Dong et al. (2019) & Gynura divaricata (L.) DC. & $\begin{array}{l}\text { Silk biotechnology Lab, Soochow University, Suzhou, } \\
\text { China }\end{array}$ & - & No & Yes-HPLC \\
\hline Ma et al. (2017) & Gynura formosana Kitam & $\begin{array}{l}\text { Plant greenhouse of Longyan University, Longyan, } \\
\text { China }\end{array}$ & - & No & No \\
\hline
\end{tabular}

Gynura bicolor (Roxb. and Willd.) DC. Gynura bicolor DC.

Gynura bicolor

nura bicolor DC.

作,

Pharmacy, Mahidol University, Bangkok, Thailand

Penang Island, Malaysia

Ethno Resources Sdn Bhd, Malaysia

Green House Facility, Faculty of Science and

Technology, Universiti Kebangsaan Malaysia (UKM)

Preve, South China

Semenyih, Selangor, Malaysia

Brightmark Sdn. Bhd, Semenyih, Malaysia

Shanxi Jintai Biol, Chin

(MARD)

Farms in Puli Town, Nanton county, Taiwan

hispida Thv

Buriram province

vera pseudochina DC. var. hispida Not stated

Gynura pseudochina (L.) DC

Koeng Sub-district, Mueang District, Maha

Sarakham province, Thailand

Gynura segetum (Lour.) Merr

Rahman et al. (2018)

ura nepalensis DC.

Gynura divaricata L

Silk biotechnology Lab, Soochow University, Suzhou,

Plant greenhouse of Longyan University, Longyan,

HPLC, high-performance liquid chromatography; +, includes a voucher specimen; -, a voucher specimen is missing. 
is a synonym of Gynura barbareifolia Gagnep and Gynura divaricata subsp. formosana (Kitam.) FG Davies is a synonym of Gynura formosana Kitam (The Plant List, 2020).

A total of 17 selected studies (Siriwatanametanon et al., 2010; Kim et al., 2011; Akowuah et al., 2012; Wu et al., 2013; Seow et al., 2014; Shwter et al., 2014; Chao et al., 2015; Wong et al., 2015; Yu et al., 2016; Yin et al., 2017; Yuandani et al., 2017; Rahman et al., 2018; Sukadeetad et al., 2018; Huang et al., 2019; Nazri et al., 2019; Ning et al., 2019; Chandradevan et al., 2020) provided the full information about the botanical material and authenticated the Gynura species by depositing voucher specimens. Six selected studies (Iskander et al., 2002; Xu et al., 2015; Ma et al., 2017; Dong et al., 2019; Liu M. et al., 2019; Liu Y. et al., 2019) reported the information of the Gynura species source but did not provide voucher specimens. Four selected studies (Siriwatanametanon and Heinrich, 2011; Rerknimitr et al., 2016; Pai et al., 2019; Yang et al., 2019) were inadequate on material information as the Gynura species source was not reported and voucher specimens were missing. The absence of voucher specimens seriously makes the reliability of the article suspicious. Sufficient description of the experimental methods used and citation of voucher specimens as evidence of the plants used are critical factors of the repeatability of the ethnopharmacological or any botanical study. Otherwise, the scientific impact of a study will be drastically diminished. Erroneous identification is a serious problem that may jeopardize any recorded uses or properties that may, in fact, relate to different species (Rivera et al., 2014).

All selected studies showed no data on quality control as none of the Gynura preparation was mentioned to follow the monograph of pharmacopeia. The safety, efficacy, and quality control of medicinal plants are getting more attention from both health authorities and the public. Herbal medicines are in widespread use, and the public believes that natural products are safe and devoid of adverse effects. However, medicinal plants are often used in combination, may be contaminated and adulterated, and may contain toxic compounds. The common misconception among the public often leads to inappropriate use and uncontrolled consumption, where poisoning and acute health problems are possible consequences. Hence, quality control of herbal medicines has a direct impact on their safety and efficacy (Wachtel-Galor and Benzie, 2011). According to good manufacturing practice, the crucial requirements for quality control of starting materials include correct identification of medicinal plant species, special storage, and special sanitation and cleaning methods for various materials (Ekor, 2014).

From harvesting to manufacturing, the quality of herbal medicines can be affected by vast factors. Quality evaluation of medicinal plants is possible by detecting the presence of chemical markers within a sample (Li et al., 2008). Qualitative chemical evaluation covers identification and characterization of phytochemical constituents in the medicinal plants through different analytical techniques. Phytochemical screening techniques involve botanical identification, extraction with suitable solvents, purification, and characterization of the active constituents of pharmaceutical importance (Folashade et al., 2012). Chromatographic methods such as thin-layer chromatography/high-performance TLC (TLC/HP-TLC) and high-performance liquid chromatography (HPLC), which are the most commonly used chemical techniques in the identification and quality assessment of medicinal plant ingredients, have provided characteristic qualitative and quantitative patterns of the constituents. Spectroscopic techniques, including UV, IR, and nuclear magnetic resonance (NMR), allow the quantitation of single or multiple compounds that share similarities in their UV absorbance, thus providing a more holistic view of herbal medicines in contrast to the quantitation of a single compound (Upton et al., 2019). Chemical analysis of Gynura species were reported in studies conducted by Kim et al. (2011), Siriwatanametanon and Heinrich (2011), Wu et al. (2013), Yu et al. (2016), Yuandani et al. (2017), Rahman et al. (2018), Sukadeetad et al. (2018), Dong et al. (2019), Huang et al. (2019), Liu M. et al. (2019), Liu Y. et al. (2019), Nazri et al. (2019), and Chandradevan et al. (2020) using methods such as HP-TLC, HPLC, gas chromatography-mass spectrometer, electrospray ionization-mass spectrometry, electrospray ionization time-of-flight mass spectrometry, liquid chromatography with tandem mass spectrometry, ultra-highperformance liquid chromatography-quadrupole time-of-flight mass spectrometry, ${ }^{1} \mathrm{H} \mathrm{N}^{13} \mathrm{C}$ NMR, and 2D-NMR.

On the basis of the risk of bias score in Table 2, under the domain "other sources of bias," five studies (Siriwatanametanon et al., 2010; Kim et al., 2011; Siriwatanametanon and Heinrich, 2011; Chao et al., 2015; Rahman et al., 2018) were rated as "definitely high" risk of bias because of the unclear or absence of statistical analysis. This finding raised the validity of the interpretations of these studies in question because the claimed significant results are possibly misinterpreted under errors of statistical analysis. Hence, the erroneous statistical methods may contribute to false positive findings and may successively become misleading literature. Under the domain "Detection bias," Iskander et al. (2002) and Rahman et al. (2018) were rated as definitely high risk of bias because the outcome assessment methods used were insensitive to indicate antioxidant or anti-inflammatory effects.

\section{Study Characteristics Species of Gynura}

Of the 27 selected studies, there are seven species of genus Gynura, which are G. procumbens, G. bicolor, G segetum, $G$. divaricata, G. formosana, G. nepalensis, and G. pseudochina. Among these Gynura species, 11 out of 27 selected studies focused on G. procumbens (Iskander et al., 2002; Kim et al., 2011; Akowuah et al., 2012; Shwter et al., 2014; Wong et al., 2015; Huang et al., 2019; Liu M. et al., 2019; Liu Y. et al., 2019; Nazri et al., 2019; Ning et al., 2019; Chandradevan et al., 2020), five studies on G. bicolor (Wu et al., 2013; Chao et al., 2015; Yin et al., 2017; Pai et al., 2019; Yang et al., 2019), four studies on G. pseudochina (Siriwatanametanon et al., 2010; Siriwatanametanon and Heinrich, 2011; Rerknimitr et al., 2016; Sukadeetad et al., 2018), two studies on G. segetum (Seow et al., 2014; Yuandani et al., 2017), two studies on G. nepalensis (Yu et al., 2016; Rahman et al., 2018), two studies on G. divaricata (Xu et al., 2015; Dong et al., 2019), and one study on G. formosana (Ma et al., 2017).

Identical plant species might exhibit phytochemical or bioactivity variations that can possibly be attributed to intrinsic factors (age of the plant and part of the plant used) and extrinsic 
TABLE 2 | Risk of bias assessment of each individual study on Gynura species according to OHAT guideline.

\begin{tabular}{|c|c|c|c|c|c|c|c|c|c|c|c|c|c|c|c|c|c|c|c|c|c|c|c|c|c|c|c|c|}
\hline Domain & Questions & $\begin{array}{l}\text { Iskander } \\
\text { et al. } \\
\text { (2002) }\end{array}$ & $\begin{array}{c}\text { Akowuah } \\
\text { et al. } \\
(2012)\end{array}$ & $\begin{array}{l}\text { Kim } \\
\text { et al. } \\
\text { (2011) }\end{array}$ & $\begin{array}{l}\text { Shwter } \\
\text { et al. } \\
(2014)\end{array}$ & $\begin{array}{l}\text { Wong } \\
\text { et al. } \\
(2015)\end{array}$ & $\begin{array}{l}\text { Huang } \\
\text { et al. } \\
(2019)\end{array}$ & $\begin{array}{l}\text { LiuM. } \\
\text { et al. } \\
\text { (2019) }\end{array}$ & $\begin{array}{l}\text { Nazri } \\
\text { et al. } \\
\text { (2019) }\end{array}$ & $\begin{array}{l}\text { Ning } \\
\text { et al. } \\
\text { (2019) }\end{array}$ & $\begin{array}{l}\text { Liu Y. } \\
\text { et al. } \\
\text { (2019) }\end{array}$ & $\begin{array}{l}\text { Chandradevan } \\
\text { et al. } \\
(2020)\end{array}$ & $\begin{array}{l}\text { Wu } \\
\text { et al. } \\
\text { (2013) }\end{array}$ & $\begin{array}{l}\text { Chao } \\
\text { et al. } \\
\text { (2015) }\end{array}$ & $\begin{array}{l}\text { Yin } \\
\text { et al. } \\
\text { (2017) }\end{array}$ & $\begin{array}{l}\text { Pai } \\
\text { et al. } \\
\text { (2019) }\end{array}$ & $\begin{array}{l}\text { Yang } \\
\text { et al. } \\
\text { (2019) }\end{array}$ & $\begin{array}{l}\text { Siriwatanametanon } \\
\text { et al. } \\
(2010)\end{array}$ & $\begin{array}{l}\text { Siriwatanametanon } \\
\text { and Heinrich } \\
\text { (2011) }\end{array}$ & $\begin{array}{l}\text { Rerknimitr } \\
\text { et al. } \\
\text { (2016) }\end{array}$ & $\begin{array}{l}\text { Sukadetad } \\
\text { et al. } \\
(2018)\end{array}$ & $\begin{array}{l}\text { Seow } \\
\text { et al. } \\
\text { (2014) }\end{array}$ & $\begin{array}{l}\text { Yuandani } \\
\text { et al. } \\
\text { (2017) }\end{array}$ & $\begin{array}{l}\text { Yu } \\
\text { et al. } \\
\text { (2016) }\end{array}$ & $\begin{array}{c}\text { Rahman } \\
\text { et al. } \\
\text { (2018) }\end{array}$ & $\begin{array}{c}\mathrm{Xu} \\
\text { et al. } \\
(2015)\end{array}$ & $\begin{array}{l}\text { Dong } \\
\text { et al. } \\
\text { (2019 }\end{array}$ & $\begin{array}{l}\text { Ma } \\
\text { et al. } \\
\text { (2017) }\end{array}$ \\
\hline \multirow[t]{2}{*}{$\begin{array}{l}\text { Selection } \\
\text { bias }\end{array}$} & $\begin{array}{l}\text { 1. Was } \\
\text { administered } \\
\text { dose or } \\
\text { exposure level } \\
\text { adequately } \\
\text { randomized? }\end{array}$ & 2 & 3 & 3 & 2 & 3 & 2 & 2 & 2 & 2 & 2 & 2 & 2 & 2 & 2 & 2 & 2 & 2 & 2 & 2 & 2 & 3 & 2 & 2 & 3 & 2 & 2 & 2 \\
\hline & $\begin{array}{l}\text { 2. Was allocation } \\
\text { to study groups } \\
\text { adequately } \\
\text { concealed? }\end{array}$ & 3 & 3 & 3 & 3 & 3 & 3 & 3 & 3 & 3 & 3 & 3 & 3 & 3 & 3 & 3 & 3 & 3 & 3 & 1 & 3 & 3 & 3 & 3 & 3 & 3 & 3 & 3 \\
\hline \multirow[t]{2}{*}{$\begin{array}{l}\text { Performance } \\
\text { bias }\end{array}$} & $\begin{array}{l}\text { 5. Were } \\
\text { experimental } \\
\text { conditions } \\
\text { identical across } \\
\text { study groups? }\end{array}$ & 2 & 2 & 3 & 1 & 1 & 2 & 3 & 2 & 1 & 2 & 2 & 2 & 3 & 1 & 2 & 1 & 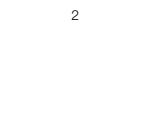 & 3 & $N R$ & 1 & 1 & 3 & 2 & 2 & 2 & 2 & 3 \\
\hline & $\begin{array}{l}\text { 6. Were the } \\
\text { research } \\
\text { personnel and } \\
\text { human subjects } \\
\text { blinded to the } \\
\text { study group } \\
\text { during the } \\
\text { study? }\end{array}$ & 3 & 3 & 3 & 3 & 3 & 3 & 3 & 3 & 3 & 3 & 3 & 3 & 3 & 3 & 3 & 3 & 3 & 3 & 1 & 3 & 3 & 3 & 3 & 3 & 3 & 3 & 3 \\
\hline $\begin{array}{l}\text { Attrition/ } \\
\text { exclusion } \\
\text { bias }\end{array}$ & $\begin{array}{l}\text { 7. Were outcome } \\
\text { data complete } \\
\text { without attrition } \\
\text { or exclusion from } \\
\text { analysis? }\end{array}$ & 3 & 3 & 3 & 3 & 3 & 3 & 3 & 3 & 3 & 3 & 3 & 3 & 3 & 3 & 3 & 3 & 3 & 3 & 3 & 3 & 3 & 3 & 3 & 3 & 3 & 3 & 3 \\
\hline \multirow[t]{2}{*}{$\begin{array}{l}\text { Detection } \\
\text { bias }\end{array}$} & $\begin{array}{l}\text { 8. Can we be } \\
\text { confident in the } \\
\text { exposure } \\
\text { characterization? }\end{array}$ & 3 & 3 & 3 & 3 & 3 & 3 & 3 & 3 & 3 & 3 & 3 & 3 & 3 & 3 & 3 & 3 & 3 & 3 & 3 & 3 & 3 & 3 & 3 & 3 & 3 & 3 & 3 \\
\hline & $\begin{array}{l}\text { 9. Can we be } \\
\text { confident in the } \\
\text { outcome } \\
\text { assessment? }\end{array}$ & 4 & 2 & 2 & 2 & 2 & 3 & 2 & 2 & 2 & 2 & 2 & 2 & 2 & 2 & 2 & 2 & . & 2 & 2 & 2 & 2 & 2 & 2 & 4 & 2 & 2 & 2 \\
\hline $\begin{array}{l}\text { Selective } \\
\text { reporting bias }\end{array}$ & $\begin{array}{l}\text { 10. Were all } \\
\text { measured } \\
\text { outcomes } \\
\text { reported? }\end{array}$ & 2 & 2 & 3 & 2 & 2 & 2 & 2 & 2 & 2 & 2 & 4 & 2 & 2 & 2 & 2 & 2 & 2 & 2 & 2 & 2 & 2 & 2 & 2 & 2 & 2 & 2 & 2 \\
\hline $\begin{array}{l}\text { Other } \\
\text { sources of } \\
\text { bias }\end{array}$ & $\begin{array}{l}\text { 11. Were there } \\
\text { no other } \\
\text { potetialia threats } \\
\text { to internal validity } \\
\text { (e.g., statistical } \\
\text { methods were } \\
\text { appropriate and } \\
\text { researchers } \\
\text { adhered to the } \\
\text { study protocol)? }\end{array}$ & 3 & 1 & 4 & 1 & 2 & 2 & 2 & 1 & 1 & 1 & 2 & 1 & 4 & 1 & 1 & 1 & 4 & 4 & 2 & 1 & 1 & 2 & 1 & 4 & 1 & 2 & 1 \\
\hline \multicolumn{29}{|c|}{$\begin{array}{l}{ }^{1} \text { Definitely low risk of bias. } \\
{ }^{2} \text { Probably low risk of bias. } \\
{ }^{3} \text { or NR Probably high risk of bias or Not Reported. } \\
{ }^{4} \text { Definitely high risk of bias. }\end{array}$} \\
\hline
\end{tabular}


factors (geographical climate, nature of soil, season, and processing methods). The study conducted by Muraina et al. (2008) proved that the same plant extract from two different geographical locations varied in their phytochemical contents, cytotoxicity, antimicrobial and antioxidant activities. G. bicolor and G. divaricata extracts derived from plants originating from Nanjing were shown to possess higher free-radical-scavenging activities as compared to other same species extracts from different China origins (Chen et al., 2015). Hence, standardization and quality control of genus Gynura are crucial to gain regulatory approval of genus Gynura preparations as nutraceuticals or therapeutic drugs. Standardization of a drug can be used as a confirmation of its identity, quality, and purity throughout all phases of its cycle (Kumari and Kotecha, 2016). Stability testing and toxicity profiles of herbal products are important parameters in improving herbal product safety.

\section{Part of Plant Used in Studies}

Twenty selected studies used the leaves of Gynura to investigate their pharmacological effects. Nazri et al. (2019) and Ning et al. (2019) used the whole plant of G. procumbens, Xu et al. (2015) and Huang et al. (2019) used the leaf and stem parts of Gynura, Iskander et al. (2002) and Dong et al. (2019) used the aerial part of Gynura, and Liu M. et al. (2019) did not mention the part of Gynura used in their study. Hence, the leaf is the dominant part of the plant used in all selected research studies. Notably, the part of the plant that is usually consumed is the leaf. However, compared to the other parts of the plant, the root extract of both G. procumbens and G. bicolor exhibited the highest phenolic content, flavonoid content, ascorbic acid content, and antioxidant capacities (Krishnan et al., 2015). Mice with intraperitoneal injection of $G$. procumbens leaf extract at $25,50,100$, and $250 \mathrm{mg} / \mathrm{kg} /$ day for four days survived until day 30 of post-injection with no signs of toxicity such as diarrhea, excess urination, and lethargy (Vejanan et al., 2012). On the basis of the study by Zahra et al. (2011) and Shwter et al. (2014), no mortality, or organ toxicity was detected in the animals that were orally administered G. procumbens leaf extract at doses of 2 and $5 \mathrm{~g} / \mathrm{kg}$ for 14 days. The study carried out by Algariri et al. (2014) demonstrated that the leaf extract of G. procumbens was safe, with no observed acute toxicity effects as there was no treatment-related mortality at $2 \mathrm{~g} / \mathrm{kg}$ throughout the 14 days observation period. Hence, the oral lethal dose $\left(\mathrm{LD}_{50}\right)$ of $G$. procumbens extract for rats was determined to be greater than $2,000 \mathrm{mg} / \mathrm{kg}$, and the acceptable daily intake was $700 \mathrm{mg} / \mathrm{kg} /$ day. The same study also showed that consumption of 250,500 , and $1,000 \mathrm{mg} / \mathrm{kg}$ of $G$. procumbens extract for 28 days did not lead to sub-chronic toxicity as no abnormal behavior, disease, or death was observed in the animal model that received the plant extract. Administration of G. procumbens leaf extract at 1,000-5,000 mg/kg did not cause mortality or significant changes in the general behavior, bodyweight, or organ gross appearance of rats (Rosidah et al., 2009). At the same time, G. procumbens and G. bicolor leaf extracts both showed a negligible level of toxicity when administered orally at 300, 2,000, and 5,000 mg/kg for 14 days (Teoh et al., 2013, 2016).

However, the use of genus Gynura requires extra caution on toxic risk because of the presence of hepatotoxic pyrrolizidine alkaloids which was determined in the leaves of G. pseudochina (Siriwatanametanon and Heinrich, 2011; Sukadeetad et al., 2018) as well as the aerial part of G. bicolor and G. divaricata (Chen et al., 2017). Hepatic injury caused by genus Gynura has been reported in few other studies. By daily administration of the root of $G$. segetum at $1.0 \mathrm{~g}$ extract $/ \mathrm{kg}$ for 40 successive days, hepatic veno-occlusive disease, also called hepatic sinusoidal obstruction syndrome (HSOS), was induced in the mouse model where liver fibrosisrelated factors and pro-inflammatory cytokines were upregulated (Zhang et al., 2019). The mouse model in which a $30 \mathrm{~g} / \mathrm{kg}$ decoction of $G$. segetum dried rhizome was administered by gavage in the morning for 30 days showed features of HSOS, including increased weight ratio of the liver and body, serum transaminase, bilirubin, decreased albumin (Chen et al., 2011), bulging abdomen, a large number of clear ascites, floating bowels, jelly omentum in the abdominal cavity, and congested and swollen or contracted and hard liver with a grainy surface and blunt edges (Zhu et al., 2011). The potential markers of hepatotoxicity induced by the decoction of G. segetum dried rhizome are verified in the animal model as differential metabolites of arginine, creatine, valine, glutamine, and citric acid, which are involved in the regulation of multiple metabolic pathways, primarily amino acid metabolism and energy metabolism (Qiu S. et al., 2018). G. segetum dried rhizome also caused liver injury by dysregulating mitochondrial ROS generation through the SIRT3-SOD2 pathway (Li et al., 2019). The roots and aerial parts of $G$. segetum were proven to contain large amounts of pyrrolizidine alkaloids, including senecionine, which were responsible for the impaired bile acid homeostasis in the animal model orally administered $1.0 \mathrm{~g} / \mathrm{kg} \mathrm{BW} \mathrm{G}$. segetum extract for $48 \mathrm{~h}$ (Xiong et al., 2018). Hence, any contraindications caused by longterm consumption of genus Gynura must be determined prior to the commercialization of genus Gynura products. Appropriate clinical studies are needed to determine the optimal efficacy and minimum toxicity of genus Gynura. The application of genus Gynura must be closely monitored in term of doses and qualities, especially the species with reported cases of hepatotoxicity.

\section{Solvents Used for Extraction}

Five studies used the methanol extracts of G. procumbens, G. pseudochina and G. segetum to investigate their antioxidant or anti-inflammatory activities in HeLa cells (Siriwatanametanon et al., 2010), human leukocytes (Siriwatanametanon et al., 2010; Yuandani et al., 2017), murine macrophages (Yuandani et al., 2017), oxidative stress model rats (Akowuah et al., 2012), and granuloma model rats (Seow et al., 2014). As a continuation study of Siriwatanametanon et al. (2010), Siriwatanametanon and Heinrich (2011) as well as Yuandani et al. (2017) isolated compounds from the methanol extract of Gynura and determined their antioxidant or antiinflammatory effects in HeLa cells or macrophages. The extraction of G. procumbens with $80 \%$ methanol at a temperature below $60^{\circ} \mathrm{C}$ would give greater retention of the total phenolic content (TPC) and greater expression of free radical scavenging activity (FRSA) (Akowuah et al., 2009). Methanol was also identified as a more effective extraction solvent of $G$. procumbens compared to $95 \%$ ethanol and water extracts based on its higher TPC and FRSA (Akowuah et al., 2012). Sukadeetad et al. (2018) demonstrated a higher chlorogenic acid content in G. pseudochina leaf extract by microwave drying as well as good efficiency for recovering phenolic compounds by extraction with $50 \%(\mathrm{v} / \mathrm{v})$ methanol. However, 
TABLE 3 | List of studies on the antioxidant effects of genus Gynura.

\begin{tabular}{|c|c|c|c|c|c|c|}
\hline Plant species & $\begin{array}{l}\text { Part, Gynura } \\
\quad \text { form }\end{array}$ & $\begin{array}{l}\text { Cell line/Animal } \\
\text { study model }\end{array}$ & $\begin{array}{l}\text { Concentration/dose, control } \\
\text { groups }\end{array}$ & $\begin{array}{l}\text { Parameter measured } \\
\text { and technique } \\
\text { used }\end{array}$ & Findings & Reference \\
\hline \multirow[t]{13}{*}{$\begin{array}{l}\text { Gynura procumbens } \\
\text { (lour.) Merr }\end{array}$} & Leaf & Human HaCaT keratinocytes & $\begin{array}{l}\text { 1, } 10,50 \mu \mathrm{g} / \mathrm{ml} \\
\text { Control groups } \\
\text { Normal control } \\
\left.\text { Model control (UV } 40 \mathrm{~mJ} / \mathrm{cm}^{2}\right)\end{array}$ & $\begin{array}{l}\text { Intracellular ROS production level by } \\
\text { dichlorofluorescein (DCF) content }\end{array}$ & $\begin{array}{l}\text { GP extract treatment inhibited UV- } \\
\text { induced ROS generation levels about } \\
36 \% \text { at } 50 \mu \mathrm{g} / \mathrm{ml}\end{array}$ & $\begin{array}{l}\text { Kim et al. } \\
\text { (2011) }\end{array}$ \\
\hline & Extract (ethanol) & $\begin{array}{l}\text { in vitro study stimulated by UV } \\
\text { irradiation }\end{array}$ & $\begin{array}{l}\text { Reference group (50, 100, } \\
200 \mu \mathrm{g} / \mathrm{ml} \text { vitamin C) }\end{array}$ & & & \\
\hline & Leaf & Mice normal liver cell line NCTC-1469 & $\begin{array}{l}80 \text { and } 160 \mu \mathrm{g} / \mathrm{ml}(24 \mathrm{~h}) \\
\text { Control groups }\end{array}$ & Intracellular ROS production level & $\begin{array}{l}\downarrow \text { ROS level by } 80 \text { and } 160 \mu \mathrm{g} / \mathrm{ml} \mathrm{GP} \\
\text { extract }\end{array}$ & $\begin{array}{l}\text { Liu Y. et al. } \\
\text { (2019) }\end{array}$ \\
\hline & Extract (aqueous) & $\begin{array}{l}\text { In vitro study stimulated by palmitic } \\
\text { acid and oleic acid }\end{array}$ & $\begin{array}{l}\text { Normal control (culture medium) } \\
\text { Model control (pre-treated with } \\
0.25 \mathrm{mM} \text { palmitic acid (PA) + } 0.5 \mathrm{mM} \\
\text { oleic acid for } 24 \mathrm{~h} \text { ) }\end{array}$ & & & \\
\hline & Leaf & 18 sprague-dawley rats (6 rats/group) & $\begin{array}{l}1.0 \mathrm{~g} / \mathrm{kg} \text { body weight } \\
\text { Oral route }\end{array}$ & $\begin{array}{l}\text { Plasma lipid peroxidation levels using } \\
\text { thiobarbituric acid reactive substances }\end{array}$ & $\downarrow$ plasma TBARS level & $\begin{array}{l}\text { Akowuah et al. } \\
\text { (2012) }\end{array}$ \\
\hline & Extract (methanol) & $\begin{array}{l}\text { In vivo study using carbon } \\
\text { tetrachloride }\left(\mathrm{CCl}_{4}\right) \text {-induced } \\
\text { oxidative stress rats }\end{array}$ & $\begin{array}{l}\text { Daily single dose for } 14 \text { days } \\
\text { Control groups } \\
\text { Normal control } \\
\text { Model control (single dose of } \mathrm{CCl}_{4} \text { ) }\end{array}$ & $\begin{array}{l}\text { (TBARS) assay } \\
\text { Plasma total antioxidant status (TAS) }\end{array}$ & $\begin{array}{l}\text { TAS values of rats fed with the } \\
\text { extract }(1 \mathrm{~g} / \mathrm{kg}) \text { for } 14 \text { days followed } \\
\text { by } \mathrm{CCl}_{4} \text { administration were } \\
\text { comparable to values of control } \\
\text { group }\end{array}$ & \\
\hline & Leaf & $\begin{array}{l}30 \text { adult male sprague-dawley rats }(6 \\
\text { rats/group) }\end{array}$ & $\begin{array}{l}250 \text { and } 500 \mathrm{mg} / \mathrm{kg} \text { body weight } \\
\text { Oral route } \\
\text { Daily for } 10 \text { weeks }\end{array}$ & $\begin{array}{l}\text { Lipid peroxidation levels using TBARS } \\
\text { assay } \\
\text { Glutathione-S-transferase (GST) } \\
\text { activity }\end{array}$ & $\begin{array}{l}\uparrow \text { GST and SOD activities in treated } \\
\text { rats }(250 \text { and } 500 \mathrm{mg} / \mathrm{kg} \text { ) }\end{array}$ & $\begin{array}{l}\text { Shwter et al. } \\
\text { (2014) }\end{array}$ \\
\hline & & & Control groups & Superoxide dismutase (SOD) activity & & \\
\hline & & $\begin{array}{l}\text { In vivo study using carcinogen- } \\
\text { induced colon cancer rats }\end{array}$ & $\begin{array}{l}\text { Normal control (normal saline } \\
\text { subcutaneous injections, 10\% tween } \\
20 \text { oral administration) } \\
\text { Carcinogen group (azoxymethane } \\
\text { (AOM) subcutaneous injection }+10 \% \\
\text { tween } 20 \text { oral administration) }\end{array}$ & & $\begin{array}{l}\downarrow \text { MDA level in treatment groups } 250 \\
\text { and } 500 \mathrm{mg} / \mathrm{kg} \text { GP. }\end{array}$ & \\
\hline & Extract (ethanol) & & $\begin{array}{l}\text { Reference group (AOM, fluorouracil } \\
\text { intraperitoneal injection) }\end{array}$ & & & \\
\hline & Whole plant & $\begin{array}{l}48 \text { female sprague-dawley rats (6 rats/ } \\
\text { group) }\end{array}$ & 250 and $500 \mathrm{mg} / \mathrm{kg}$ body weight & $\begin{array}{l}\text { Plasma malondialdehyde (MDA) level } \\
\text { using high-performance liquid } \\
\text { chromatography (HPLC) }\end{array}$ & $\begin{array}{l}\downarrow \text { MDA level in treatment groups } 250 \\
\text { and } 500 \mathrm{mg} / \mathrm{kg} \text { GP compared to } \\
\text { postmenopausal control group at } \\
\text { month } 3 \text { and month } 6\end{array}$ & $\begin{array}{l}\text { Nazri et al. } \\
\text { (2019) }\end{array}$ \\
\hline & Extract (ethanol) & & Oral route & & $\uparrow \mathrm{SOD}, \mathrm{GSH}-\mathrm{Px}$ and CAT enzyme & \\
\hline & & $\begin{array}{l}\text { In vivo study using postmenopausal } \\
\text { rats fed with cholesterol diet } \\
\text { enriched with repeatedly heated } \\
\text { palm oil }\end{array}$ & $\begin{array}{l}\text { Daily for } 24 \text { weeks } \\
\text { Control groups } \\
\text { Sham group (rats received basal diet) } \\
\text { Postmenopausal (PM) group (rats } \\
\text { subjected to ovariectomy, received } 2 \% \\
\text { cholesterol diet fortified with five-time } \\
\text { heated palm oil (5HPO) } \\
\text { Positive control (10 mg/kg atorvastatin) }\end{array}$ & $\begin{array}{l}\text { Superoxide dismutase (SOD) activity } \\
\text { Glutathione peroxidase (GSH-Px) } \\
\text { activity } \\
\text { catalase (CAT) activity }\end{array}$ & $\begin{array}{l}\text { activities in treated rats ( } 250 \text { and } \\
500 \mathrm{mg} / \mathrm{kg} \text { ) compared to } \\
\text { postmenopausal control group. GP } \\
\text { maintained SOD activity from month } \\
3 \text { to month } 6\end{array}$ & \\
\hline
\end{tabular}


TABLE 3 | (Continued) List of studies on the antioxidant effects of genus Gynura.

$\begin{array}{ccc}\text { Plant species } & \begin{array}{c}\text { Part, Gynura } \\ \text { form }\end{array} & \begin{array}{c}\text { Cell line/Animal } \\ \text { study model }\end{array}\end{array}$

Concentration/dose, control

groups

\section{Parameter measured}

and technique

used
Extract (aqueous)

Leaf

Gynura bicolor (roxb. Leaf

Ex willd.) DC.

Extract (aqueous, ethanol)

Leaf

Extract (aqueous)

Leaf

Extract (aqueous)

vivoA

Leaf

Extract (aqueous)

Gynura divaricata (L.) Leaf, stem

DC.

Lyophilized into powder
32 male C57BL/6 J mice (8 mice/

group

In vivo study using non-alcoholic steatohepatitis (NASH) mice

Oral route

Daily for 6 weeks

Control groups

Normal control (methionine- and

choline-sufficient (MCS) diet)

Model control (methionine- and

choline-deficient (MCD) diet)

Human umbilical vein endothelial cells Aqueous or ethanol extract at 1,2 or $4 \%$ ROS production level by $2^{\prime}, 7^{\prime}$ -

$12 \mathrm{~h}$ pre-treatment

In vitro study treated by high glucose Control groups

Control (5.5 mM glucose)

Model control (33 mM glucose)

PC12 cell line (rat adrenal gland $\quad$ Aqueous extract at $0.25,0.5$ or $1 \%$

pheochromocytoma) $\quad 48 \mathrm{~h}$ pre-treatment

Control groups

Normal control

In vitro study of $\mathrm{H}_{2} \mathrm{O}_{2}$ induced injury Model control $\left(\mathrm{H}_{2} \mathrm{O}_{2}\right.$ stimulation)

C57BL6 mice (8 mice/group) $\quad 0.25$ or $0.5 \%$ G. bicolor aqueous extra

In vivo study using chronic ethanol diet

consumption-induced hepatic injury Oral route

mice model

6 weeks

Control groups

Normal control

Liquid diet group (without ethano)

Ethanol diet group

50 male Balb/cA mice (10 mice/group) $\quad 0.25,0.5,1 \%$ GB diet

Oral route

Control groups

Normal control (standard mouse basal

In vivo streptozotocin-induced type

1 diabetic mice model

Diabetic model control $(40 \mathrm{mg} / \mathrm{kg}$ BW streptozotocin via i.p. injection for 5 days)

60 male imprinting control region mice Diets with $1.2 \%$ and $4.8 \% \mathrm{GD}$

(15 mice/group)

Oral route

In vivo study using high-fat diet and Daily for 4 weeks

streptozotocin (STZ) induced type 2 Control groups

diabetic mice
Hepatic malondialdehyde (MDA) level $\quad \downarrow \downarrow$ MDA level in treatment groups $\quad$ Liu Y. et al.

Glutathione peroxidase (GSH-Px) 500 and $1,000 \mathrm{mg} / \mathrm{kg} \mathrm{GP}$.

$\uparrow \uparrow$ GSH-Px, CAT and HO- 1 activities

in treated mice $(500$ and

catalase (CAT) activity

Heme oxygenase 1 (HO-1) activity $1,000 \mathrm{mg} / \mathrm{kg}$ )

dichlorofluorescein (DCF) content

catalase (CAT) activity

Pre-treatments with aqueous or Chao et al.

ethanol extract dose-dependently $\downarrow$ (2015)

ROS level and preserved GSH

Glutathione (GSH) content Pre-treatment at 2 and $4 \%$ retained

Glutathione peroxidase activity (GSH- CAT and GSH-Px activities

Px)

ROS level by $2^{\prime}, 7^{\prime}$-Dichlorofluorescein GB pre-treatments $\uparrow$ GSH content, $\uparrow$ Yang et al.

diacetate (DCFH-DA) GSH-Px activity, concentration-

dependently $\downarrow$ ROS level and $\uparrow$

Glutathione level (GSH) $\quad$ dependently $\downarrow$

$\mathrm{Px}$ )

CAT activity assay

Hepatic GSH or GSSG content

$0.25 \%$ or $0.5 \%$ GB $\uparrow$ GSH content, $\downarrow$ Yin et al. (2017) Glutathione peroxidase activity (GSH- GSSG, $\downarrow$ ROS levels, maintained

Glutathione reductase (GR) and

catalase

Hepatic ROS level using DCFH-DA

ROS level by DCFH-DA

Glutathione level (GSH)

GB at 3 doses

Pai et al. (2019)

Glutathione peroxidase activity (GSH- $\uparrow$ GSH content, $\uparrow$ GPX $\uparrow$ GR, and $\uparrow$

Glutathione reductase (GR)

catalase activities $\downarrow R O S$ level in heart

CAT activity assay

Px)

MDA level $4.8 \%$ GD

(SOD) MDA leve

Lipid peroxidation level by MDA level

Ax level, $\uparrow$ T-SOD level,

assay
Diabetic model control (high-fat diet

(18\% lard, 20\% sugar, 3\% egg yolk,

$59 \%$ basal diet) and $100 \mathrm{mg} / \mathrm{kg}$ STZ 
TABLE 3 | (Continued) List of studies on the antioxidant effects of genus Gynura.

\begin{tabular}{|c|c|c|c|c|c|c|}
\hline Plant species & $\begin{array}{l}\text { Part, Gynura } \\
\text { form }\end{array}$ & $\begin{array}{l}\text { Cell line/Animal } \\
\text { study model }\end{array}$ & $\begin{array}{c}\text { Concentration/dose, control } \\
\text { groups }\end{array}$ & $\begin{array}{l}\text { Parameter measured } \\
\text { and technique } \\
\text { used }\end{array}$ & Findings & Reference \\
\hline & $\begin{array}{l}\text { Aerial part } \\
\text { Lyophilized into powder }\end{array}$ & $\begin{array}{l}\text { Male ICR mice ( } 15 \text { mice/group) } \\
\text { In vivo study using high-fat diet and } \\
\text { streptozotocin (STZ) induced type } 2 \\
\text { diabetic mice }\end{array}$ & $\begin{array}{l}\text { Diets with } 1 \%, 5 \% \text { and } 10 \% \text { GD } \\
\text { Oral route } \\
\text { Daily for } 4 \text { weeks } \\
\text { Control groups } \\
\text { Normal control (normal chow) } \\
\text { Model control (high-fat diet (18\% lard, } \\
20 \% \text { sugar, } 3 \% \text { egg yolk and } 59 \% \\
\text { basal diet) and } 100 \mathrm{mg} / \mathrm{kg} \mathrm{STZ)}\end{array}$ & $\begin{array}{l}\text { Hepatic glutathione peroxidase activity } \\
\text { (GSH-Px) } \\
\text { Hepatic total superoxide dismutase } \\
\text { activity (SOD) } \\
\text { Hepatic lipid peroxidation level by } \\
\text { MDA level assay } \\
\text { Hepatic 8-hydroxy-2'- } \\
\text { deoxyguanosine (8-OHdG) level using } \\
\text { ELISA }\end{array}$ & $\begin{array}{l}\text { 1\% GD: } \uparrow \text { GSH-Px level, } \uparrow \text { T-SOD } \\
\text { level } \\
\text { 5\% GD: } \uparrow \text { GSH-Px level, } \uparrow \uparrow \text { T-SOD } \\
\text { level, } \downarrow \text { 8-OHdG level } \\
\text { 10\% GD: } \uparrow \uparrow \text { GSH-Px level, } \uparrow \text { T-SOD } \\
\text { level, } \downarrow \text { MDA level, } \downarrow \text { 8-OHdG level }\end{array}$ & $\begin{array}{l}\text { Dong et al., } \\
(2019)\end{array}$ \\
\hline $\begin{array}{l}\text { Gynura segetum } \\
\text { (lour.) Merr }\end{array}$ & $\begin{array}{l}\text { Extract (methanol), isolated } \\
\text { compounds from extract }\end{array}$ & $\begin{array}{l}\text { In vitro study induced by opsonized } \\
\text { zymosan or PMA }\end{array}$ & $\begin{array}{l}\text { Extract: } 6.25-100 \mu \mathrm{g} / \mathrm{ml} \\
\text { Compounds: } 3.125-50 \mu \mathrm{g} / \mathrm{ml} \\
\text { Control groups } \\
\text { Negative control (without sample) } \\
\text { Positive control (acetylsalicylic acid) }\end{array}$ & $\begin{array}{l}\text { ROS production level by luminol } \\
\text { method }\end{array}$ & $\begin{array}{l}\text { GS extract exhibited inhibitory } \\
\text { activity upon activation by PMA } \\
\left(\mathrm{I} \mathrm{C}_{50}=1.41 \pm 0.63 \mu \mathrm{g} / \mathrm{ml}\right) \text { and } \\
\text { zymosan }\left(\mathrm{IC}_{50}=2.63 \pm 0.89\right. \\
\mu \mathrm{g} / \mathrm{ml}) \\
8,8^{\prime} \text {-(ethene-1,2-diyl)-dinaphtalene- } \\
1,4,5 \text {-triol revealed ROS inhibitory } \\
\text { upon activation by PMA }\left(\mathrm{IC}_{50}=\right. \\
0.13 \mu \mathrm{M}) \text { and zymosan }\left(\mathrm{I} \mathrm{C}_{50}=\right. \\
0.05 \mu \mathrm{M}) \\
\text { Rutin inhibited ROS activated by } \\
\text { PMA }\left(\mathrm{I} \mathrm{C}_{50}=0.08 \mu \mathrm{M}\right) \text { and zymosan } \\
\left(\mathrm{I} \mathrm{C}_{50}=0.13 \mu \mathrm{M}\right)\end{array}$ & $\begin{array}{l}\text { Yuandani et al. } \\
\text { (2017) }\end{array}$ \\
\hline $\begin{array}{l}\text { Gynura nepalensis } \\
\text { DC. }\end{array}$ & $\begin{array}{l}\text { Nine caffeoylquinic acid analogs } \\
\text { (1-9) isolated from ethanol extract }\end{array}$ & In vitro study stimulated by $\mathrm{H}_{2} \mathrm{O}_{2}$ & $\begin{array}{l}0.78,1.56,3.12,6.25,12.5,25,50, \\
100 \mu \mathrm{M} \\
1 \mathrm{~h} \text { pre-treatment } \\
\text { Control groups } \\
\text { Normal control } \\
\text { Model control }\left(0.3 \mathrm{mM} \mathrm{H}_{2} \mathrm{O}_{2}\right)\end{array}$ & $\begin{array}{l}\text { Intracellular ROS production by DCF } \\
\text { content } \\
\text { CAT activity assay }\end{array}$ & $\begin{array}{l}\text { Compound } 6 \text { ( } 3,5 \text {-dicaffeoylquinic } \\
\text { acid ethyl ester) exhibited a more } \\
\text { potent cytoprotective effect thus } \\
\text { selected for further evaluation } \\
\downarrow \downarrow \downarrow R O S \text { production in } \mathrm{H}_{2} \mathrm{O}_{2} \text {-treated } \\
\text { cells, even at a concentration of } \\
0.78 \mu \mathrm{M} \\
\mu_{1} \\
\text { activity at doses of } 25 \text { and } 50 \mu \mathrm{M}\end{array}$ & Yu et al. (2016) \\
\hline $\begin{array}{l}\text { Gynura formosana } \\
\text { kitam }\end{array}$ & Extract (ethyl acetate) & $\begin{array}{l}48 \text { male sprague-dawley rats (8 rats/ } \\
\text { group) } \\
\text { In vivo study using cotton pellet- } \\
\text { induced granuloma rat model }\end{array}$ & $\begin{array}{l}100 \mathrm{mg} / \mathrm{kg}, 250 \mathrm{mg} / \mathrm{kg}, 500 \mathrm{mg} / \mathrm{kg} \\
\text { body weight } \\
\text { Oral route } \\
\text { Once daily for } 7 \text { days } \\
\text { Control groups } \\
\text { Normal control } \\
\text { Model control ( } 0.5 \% \\
\text { carboxymethylcellulose, } 1 \mathrm{ml} / \mathrm{kg}) \\
\text { Standard drug ( } 4 \mathrm{mg} / \mathrm{kg} \text { indomethacin) }\end{array}$ & $\begin{array}{l}\text { CAT activity assay } \\
\text { Total superoxide dismutase activity } \\
\text { (SOD) } \\
\text { Glutathione level (GSH) } \\
\text { Lipid peroxidation level }\end{array}$ & $\begin{array}{l}250 \text { and } 500 \mathrm{mg} / \mathrm{kg} \text { GF treatment } \uparrow \uparrow \\
\text { activities of CAT, } \uparrow \uparrow \mathrm{SOD}, \uparrow \uparrow \mathrm{GSH} \\
\text { and } \downarrow \downarrow \text { lipid peroxidation in rat liver }\end{array}$ & Ma et al. (2017) \\
\hline
\end{tabular}

$\uparrow$ indicates significantly induce $(\mathrm{p}<0.05)$, $\uparrow \uparrow$ indicates significantly induce $(\mathrm{p}<0.01)$, $\uparrow \uparrow \uparrow$ indicates significantly induce $(\mathrm{p}<0.001), \downarrow$ indicates significantly inhibit $(\mathrm{p}<0.05)$, $\downarrow \downarrow$ indicates significantly inhibit $(\mathrm{p}<0.01)$, and $\downarrow \downarrow \downarrow$ indicates significantly inhibit $(\mathrm{p}<0.001)$. 
ethanol extract was used in this study for successive bioassays by considering ethanol as a safer solvent for health product application.

Eleven studies tested the antioxidant or anti-inflammatory activities of the ethanol extract of G. procumbens, G. bicolor, G. pseudochina and G. nepalensis in human keratinocytes (Kim et al., 2011; Sukadeetad et al., 2018), human dermal fibroblasts (Kim et al., 2011), human endothelial cells (Chao et al., 2015), murine macrophages (Chandradevan et al., 2020; Liu M. et al., 2019; Ning et al., 2019), ear-inflamed mice (Iskander et al., 2002; Rahman et al., 2018), rat colon cancer model (Shwter et al., 2014), parasite-infected mice (Wong et al., 2015), and hypercholesterolemic rats (Nazri et al., 2019). Liu M. et al. (2019) simultaneously studied the anti-inflammatory effect of the ethanol extract and its fractions, including the petroleum ether fraction, ethyl acetate fraction, $n$-butanol fraction, and water fraction. Yu et al. (2016) studied the antioxidant and anti-inflammatory effects of nine caffeoylquinic acid analogs isolated from the ethanol extract on cardiomyoblasts. The optimal extraction of $G$. divaricata was determined with $45 \%$ ethanol for $30 \mathrm{~min}$ at $90^{\circ} \mathrm{C}$, where the increase in the extraction temperature (from 40 to $100^{\circ} \mathrm{C}$ ) led to a significant elevation of the TFC, TPC, and FRSA (Wan et al., 2011). Another study on the extraction method optimization of G. bicolor showed that $40 \%$ ethanol, $40^{\circ} \mathrm{C}, 30 \mathrm{~min}$ sonication time, and 50:1 liquid-to-solid ratio were the optimal conditions for a higher extraction yield of TPC (Qiu X. L. et al., 2018).

Five studies used the aqueous extract of G. procumbens and $G$. bicolor to determine their antioxidant or anti-inflammatory activities in human endothelial cells (Chao et al., 2015), murine adrenal gland pheochromocytoma (Yang et al., 2019), murine hepatocytes (Liu Y. et al., 2019), diabetic mice (Pai et al., 2019), and liver-injured mice (Yin et al., 2017; Liu Y. et al., 2019). The ethyl acetate extract of $G$. formosana was studied for its antioxidant and anti-inflammatory activities in granuloma model rats (Ma et al., 2017). Meanwhile, the ether extract of G. bicolor was studied for its anti-inflammatory activity in murine macrophages (Wu et al., 2013). G. divaricata was lyophilized into powder and tested in diabetic mice (Xu et al., 2015; Dong et al., 2019). The study by Huang et al. (2019) determined the anti-inflammatory effect of $G$. procumbens essential oil and its active ingredients in murine macrophage and ear-inflamed mice. One randomized controlled study on patients with moderate plaque psoriasis was conducted to study the anti-inflammatory effect of G. pseudochina ointment from an ethanol extract with a ratio of one extract to 10 vehicles (Rerknimitr et al., 2016).

\section{Antioxidant Parameters}

In general, ROS production level was the major parameter measured in vitro antioxidant studies (Kim et al., 2011; Chao et al., 2015; Yu et al., 2016; Yuandani et al., 2017; Liu Y. et al., 2019; Yang et al., 2019). The other in vitro parameters included glutathione (GSH) content and GSH peroxidase (GSH-Px) or catalase (CAT) activity (Chao et al., 2015; Yu et al., 2016; Yang et al., 2019). The main focus of in vivo antioxidant studies of Gynura species was lipid peroxidation (Akowuah et al., 2012; Shwter et al., 2014; Xu et al., 2015; Ma et al., 2017; Dong et al., 2019; Liu Y. et al., 2019; Nazri et al., 2019). The other in vivo parameters measured were plasma total antioxidant status (TAS) (Akowuah et al., 2012), CAT activity (Ma et al., 2017; Liu Y. et al., 2019; Nazri et al., 2019; Pai et al., 2019), ROS production (Yin et al., 2017; Pai et al., 2019), superoxide dismutase (SOD) activity (Shwter et al., 2014; Xu et al., 2015; Ma et al., 2017; Dong et al., 2019; Nazri et al., 2019), heme oxygenase 1 (HO-1) (Liu Y. et al., 2019), 8-hydroxy-2' -deoxyguanosine (8-OHdG) level (Dong et al., 2019), and GSH-related parameters, including GSHPx activity (Xu et al., 2015; Yin et al., 2017; Dong et al., 2019; Liu Y. et al., 2019; Nazri et al., 2019; Pai et al., 2019), GSH-S-transferase (GST) activity (Shwter et al., 2014), GSH reductase (GR) activity (Yin et al., 2017; Pai et al., 2019), GSH disulfide (GSSG) content (Yin et al., 2017), and GSH content (Ma et al., 2017; Yin et al., 2017; Pai et al., 2019).

\section{Anti-inflammatory Parameters}

Overall, in vitro anti-inflammatory studies on Gynura species focused on the secretion or expression of pro-inflammatory mediators, including interleukin-1 (IL-1) (Siriwatanametanon et al., 2010; Yuandani et al., 2017; Yang et al., 2019), IL-6 (Siriwatanametanon et al., 2010; Kim et al., 2011; Chao et al., 2015; Liu M. et al., 2019; Yang et al., 2019), IL-8 (Kim et al., 2011; Sukadeetad et al., 2018), tumor necrosis factor alpha (TNF- $\alpha$ ) (Siriwatanametanon et al., 2010; Chao et al., 2015; Yuandani et al., 2017; Liu M. et al., 2019; Yang et al., 2019), prostaglandin $\mathrm{E}_{2}\left(\mathrm{PGE}_{2}\right)$ (Siriwatanametanon et al., 2010; Wu et al., 2013; Chao et al., 2015), cyclooxygenase-2 (COX-2) (Wu et al., 2013; Chao et al., 2015), inducible nitric oxide synthase (iNOS) (Wu et al., 2013; Ning et al., 2019), nitric oxide (NO) (Wu et al., 2013; Yuandani et al., 2017; Liu M. et al., 2019; Ning et al., 2019; Chandradevan et al., 2020), lactate dehydrogenase (LDH) activity, mitochondrial membrane potential ( $\Delta \psi \mathrm{m}$ ) (Yu et al., 2016; Yang et al., 2019), matrix metalloproteinase-1 and 9 (MMP-1 and MMP-9) (Kim et al., 2011), and infiltration of inflammatory cells (Huang et al., 2019). Moreover, in vitro studies included investigations on the anti-inflammatory effect of the Gynura species on the nuclear factor erythroid 2-related factor 2 (Nrf2) signaling pathway (Liu Y. et al., 2019), nuclear factor kappa B (NF-кB) signaling pathway (Siriwatanametanon et al., 2010; Siriwatanametanon and Heinrich, 2011; Wu et al., 2013; Sukadeetad et al., 2018; Yang et al., 2019), and mitogen-activated protein kinases (MAPK) signaling pathway, including c-Jun N-terminal kinase (JNK) (Yu et al., 2016; Liu Y. et al., 2019), p38 (Yu et al., 2016; Yang et al., 2019), and extracellular signal-regulated kinase (ERK) (Yu et al., 2016). Similarly, the in vivo studies also measured the parameters of IL-1 (Seow et al., 2014; Ma et al., 2017; Yin et al., 2017; Pai et al., 2019), IL-6 (Yin et al., 2017; Pai et al., 2019), and TNF-a (Seow et al., 2014; Wong et al., 2015; Ma et al., 2017; Yin et al., 2017; Dong et al., 2019; Pai et al., 2019). The other antiinflammatory effect parameters of in vivo studies were interferon- $\gamma$ $($ IFN- $\gamma$ ), IL-10, phosphorylation of glycogen synthase kinase-3 (GSK3 $\beta$ (Wong et al., 2015; Dong et al., 2019), LDH, alanine aminotransferase (GPT), c-reactive protein (CRP) (Ma et al., 2017), Nrf2 (Liu Y. et al., 2019), peroxisome proliferator-activated receptor gamma (PPAR $\gamma$ ) (Dong et al., 2019; Liu Y. et al., 2019), COX-2 (Huang et al., 2019), inflamed-ear thickness (Iskander et al., 2002; Rahman et al., 2018; Huang et al., 2019), cotton pellet granuloma (Seow et al., 2014; Ma et al., 2017), paw edema (Rahman et al., 2018; Huang et al., 2019), signaling pathways of phosphatidylinositol 3-kinase (PI3K)/protein kinase B (Akt) (Xu et al., 2015; Dong et al., 2019), JNK (Liu Y. et al., 2019), p38 (Pai et al., 
2019), and NF-кB (Dong et al., 2019; Pai et al., 2019). The effect of Gynura on NF-кB phosphorylation was also studied in a randomized controlled study (Rerknimitr et al., 2016).

\section{Antioxidant Effects of Genus Gynura}

Table 3 shows the list of studies on the antioxidant effects of genus Gynura. Several potential mechanisms for the antioxidant activity of Gynura are suggested as follows: inhibition of ROS, inhibition of lipid peroxidation, modulation of enzymatic antioxidant production or activities, and modulation of GSHrelated parameters. Figure 2 illustrates the proposed signaling pathways of antioxidant effects by Gynura species.

\section{Gynura Inhibits Reactive Oxygen Species}

At physiological concentrations, ROS is involved in many cellular activities, including gene transcription, signaling transduction, and immune response. However, overproduction of ROS can result in oxidative damage to biomolecules, including lipids, proteins, and DNA, which is the underlying cause of various diseases (Liu et al., 2018). Inhibition of ROS production using G. procumbens extracts was determined in human $\mathrm{HaCaT}$ keratinocytes at $50 \mu \mathrm{g} / \mathrm{ml}$ (inhibitory effect comparable to $200 \mu \mathrm{g} / \mathrm{ml}$ vitamin C) (Kim et al., 2011) and in murine hepatocytes at 80 and $160 \mu \mathrm{g} / \mathrm{ml}$ (Liu Y. et al., 2019); that of G. bicolor in human umbilical vein endothelial cell (huvec) at $1-4 \% \mathrm{v} / \mathrm{v}$ (Chao et al., 2015), in adrenal gland pheochromocytoma in a concentration dependent manner (Yang et al., 2019), and in liver-injured mice at 0.25 and $0.5 \%$ diet (Yin et al., 2017); and that of $G$. segetum $\left(\mathrm{IC}_{50}\right.$ for zymosan $=2.63 \pm$ $0.89 \mu \mathrm{g} / \mathrm{ml} ; \quad \mathrm{IC}_{50}$ for $\left.\mathrm{PMA}=1.41 \pm 0.63 \mu \mathrm{g} / \mathrm{ml}\right)$ in polymorphonuclear cells (PMNs) neutrophils. The compound isolated from $G$. segetum extract, $8,8^{\prime}$-(ethene-1,2-diyl)dinaphtalene-1,4,5-triol, possessed ROS inhibitory effect upon the activation by PMA $\left(\mathrm{IC}_{50}=0.13 \mu \mathrm{M}\right)$ and zymosan $\left(\mathrm{IC}_{50}=0.05 \mu \mathrm{M}\right)$. At the same time, rutin, which was also isolated from $G$. segetum extract, inhibited the ROS activated by PMA $\left(\mathrm{IC}_{50}=0.08 \mu \mathrm{M}\right)$ and zymosan $\left(\mathrm{IC}_{50}=0.13 \mu \mathrm{M}\right)$. Both isolated compounds from $G$. segetum showed a higher antioxidant effect than the positive control aspirin (Yuandani et al., 2017). One of the compounds isolated from $G$. nepalensis extract, 3,5-dicaffeoylquinic acid ethyl ester, showed an inhibitory effect on intracellular ROS production in cardiomyoblasts at a concentration of $0.78 \mu \mathrm{M}$ and above (Yu et al., 2016). In nuclear and mitochondrial DNA, the interaction of the hydroxyl radical $(\mathrm{HO} \bullet$ ) with the nucleobases of the DNA strand will produce 8 -hydroxy-2'-deoxyguanosine (8-OHdG), a predominant form of a free radical-induced oxidative lesion, which is a critical biomarker of oxidative stress (Valavanidis et al., 2009). G. divaricata diet (5 and 10\%) showed the inhibition of the hepatic 8-OHdG level in a diabetic mice model (Dong et al., 2019). G. procumbens also improved the plasma TAS in rats with induced oxidative stress (Akowuah et al., 2012).

\section{Gynura Inhibits Lipid Peroxidation}

Lipid peroxidation is a reaction of oxygen with unsaturated lipids that successively results in the production of oxidation products, including lipid peroxyl radicals and hydroperoxides. Among a wide variety of oxidation products from lipid peroxidation, malondialdehyde (MDA) appears to be the most mutagenic product (Ayala et al., 2014). G. procumbens, G. divaricata, and G. formosana exhibited inhibition of lipid peroxidation by lowering the MDA level in carbon tetrachloride-induced oxidative stress rats (Akowuah et al., 2012), carcinogen-induced colon cancer rats (Shwter et al., 2014), high-fat-diet and streptozotocin (STZ)induced diabetic mice (Xu et al., 2015; Dong et al., 2019), cotton pellet-induced granuloma rats (Ma et al., 2017), postmenopausal rats fed with cholesterol diet enriched with repeatedly heated palm oil (Nazri et al., 2019), and non-alcoholic steatohepatitis mice (Liu Y. et al., 2019). The reduction in the MDA level of G. procumbens extract-treated rats was comparable with treatments of anticancer drug, 5-fluorouracil (Shwter et al., 2014), and lipid-lowering drug atorvastatin (Nazri et al., 2019). It is worth noticing that Xu et al. (2015) and Dong et al. (2019) both studied on G. divaricata using identical treatment methods and diabetic mice models. The results of the study by Xu et al. (2015) showed a significant inhibitory effect of $G$. divaricata lyophilized powder on lipid peroxidation at treatment doses as low as $1.2 \%$ of diet. However, only $10 \%$ lyophilized powder diet was able to exert significant inhibition of lipid peroxidation in the study by Dong et al. (2019). This variation between findings could be due to the phytochemical or bioactivity variations that possibly correlated to intrinsic factors (age of the plant and part of the plant used) and extrinsic factors (geographical climate, nature of soil, season, and processing methods).

\section{Gynura Modulates Enzymatic Antioxidant Production or Activities}

The antioxidant mechanism of heme oxygenase-1 (HO-1) is found to be associated with an increase in superoxide dismutase (SOD) and CAT (Turkseven et al., 2005). HO-1 is responsible for the oxidative cleavage of heme groups, which generates biliverdin, carbon monoxide, and ferrous iron. Biliverdin is converted to bilirubin, and both of these bile pigments are potent scavengers of singlet oxygen (Stocker et al., 1990). Oral administration of G. procumbens extract was shown to increase HO-1 activity in liver-injured mice model (Liu Y. et al., 2019). SOD is an antioxidant enzyme that is responsible for the catalytical conversion of the superoxide radical $\left({ }^{*} \mathrm{O}_{2}\right)$ or singlet oxygen radical $\left({ }^{1} \mathrm{O}_{2}{ }^{-}\right)$to hydrogen peroxide $\left(\mathrm{H}_{2} \mathrm{O}_{2}\right)$ and molecular oxygen $\left(\mathrm{O}_{2}\right)$. However, the accumulation of $\mathrm{H}_{2} \mathrm{O}_{2}$ causes toxicity to body tissues or cells. Successively, CAT breaks down $\mathrm{H}_{2} \mathrm{O}_{2}$ into water and molecular oxygen, thus minimizing free radicalinduced damage (Ighodaro and Akinloye, 2018). G. procumbens, G. divaricata, and $G$. formosana showed an antioxidant effect by inducing in vivo total SOD activity (Shwter et al., 2014; Xu et al., 2015; Ma et al., 2017; Dong et al., 2019; Nazri et al., 2019). The in vitro CAT activity was induced by G. bicolor (Chao et al., 2015; Yang et al., 2019) and a compound isolated from $G$. nepalensis, which were 25 and $50 \mu \mathrm{M}$ of 3,5-dicaffeoylquinic acid ethyl ester (Yu et al., 2016). At the same time, in vivo CAT activity was increased by G. procumbens (Liu Y. et al., 2019; Nazri et al., 2019), G. bicolor (Pai et al., 2019), and G. formosana (Ma et al., 2017). Notably, the induction of SOD and CAT by the G. procumbens extract was comparable to the effects of atorvastatin (Nazri et al., 2019).

Gynura Modulates Glutathione-Related Parameters

Owing to the absence of CAT in the mitochondria, the reduction of $\mathrm{H}_{2} \mathrm{O}_{2}$ and lipid peroxides is carried out by $\mathrm{GSH}$ peroxidase 
(GSH-Px). Therefore, protection of cells against oxidative stress is reinforced by increasing the activity of GSH-Px, which plays a crucial role in inhibiting the lipid peroxidation process (Ighodaro and Akinloye, 2018). GSH-Px activity was induced by $G$. procumbens (Liu Y. et al., 2019; Nazri et al., 2019), G. bicolor (Chao et al., 2015; Yin et al., 2017; Pai et al., 2019; Yang et al., 2019), and G. divaricata (Xu et al., 2015; Dong et al., 2019). G. procumbens showed higher efficacy in inducing GSH-Px activity as compared to atorvastatin (Nazri et al., 2019). GSH is a crucial lowmolecular-weight antioxidant that protects cells from oxidative damage via reduction, conjugation, and interaction with other non-enzymatic antioxidants (Forman et al., 2009). G. formosana extract diet (250 and $500 \mathrm{mg} / \mathrm{kg}$ ) was found to increase hepatic GSH level in granuloma rat model (Ma et al., 2017), and G. bicolor extract also showed in vitro and in vivo preservation on GSH content (Chao et al., 2015; Yin et al., 2017; Pai et al., 2019; Yang et al., 2019). GSH reductase (GR) is vital in maintaining the supply of reduced GSH, and GSH disulfide (GSSG) indicates the level of oxidized GSH (Chakravarthi et al., 2006). G. bicolor extract (0.25, 0.5, and $1 \%$ of diet) maintained GR activity (Yin et al., 2017; Pai et al., 2019) and suppressed the hepatic GSSG content in the liver-injured mice model (Yin et al., 2017). GSH-S-transferase (GST) exerted protection on cellular macromolecules from damage of reactive electrophiles by catalyzing the conjugation of GSH to various endogenous and exogenous electrophilic compounds (Townsend and Tew, 2003). G. procumbens extract diet showed antioxidant potential by increasing in vivo GST activity (Shwter et al., 2014).

\section{Anti-inflammatory Effects of Genus Gynura}

Table 4 shows the studies on the anti-inflammatory effects of Gynura. The potential mechanisms for the anti-inflammatory activity of Gynura are suggested as follows: modulation of inflammatory cytokine production, inhibition of $\mathrm{PGE}_{2}$ and $\mathrm{NO}$ production, inhibition of cellular inflammatory-related parameters, and inhibition of inflammation on animal models. As shown in Figure 2, the several potential anti-inflammatory signaling pathways of Gynura are PI3K/Akt, Nrf2, PPAR $\gamma$, GSK3, NF-кB, and MAPK.

Gynura Modulates Inflammatory Cytokine Production Cytokines are released by cells for signaling, where critical proinflammatory cytokines, including IL-1, IL-6, IL-8, IFN- $\gamma$, and $\mathrm{TNF}_{-} \alpha$, are involved in the upregulation of inflammatory reactions (Turner et al., 2014) and IL-10 is a potent anti-inflammatory cytokine (Zhang and An, 2007). G. procumbens inhibited the IL-6 and IL-8 production in UV-induced HaCaT keratinocytes (Kim et al., 2011). G. procumbens extract also dose-dependently suppressed the IL-6 production in macrophages and inhibited the TNF- $\alpha$ level. At the same time, the ethyl acetate fraction of $G$. procumbens showed the best inhibitory effect on IL-6 and TNF- $\alpha$ (Liu M. et al., 2019). In a parasite-infected mice model, G. procumbens reduced the levels of the liver and serum TNF- $\alpha$ and IFN- $\gamma$ as well as increased IL-10 level (Wong et al., 2015). G. bicolor possessed an anti-inflammatory effect by inhibiting the production of IL- $1 \beta$, IL- 6 , and TNF- $\alpha$ both in vitro (Chao et al., 2015; Yang et al., 2019) and in vivo (Yin et al., 2017; Pai et al., 2019). G. pseudochina var. hispida methanol extract also caused inhibition of IL- $1 \beta$ production $\left(\mathrm{IC}_{50}=2.46 \mu \mathrm{g} / \mathrm{ml}\right)$. Meanwhile, ethyl acetate extract of G. pseudochina var. hispida showed strong inhibition of the release of IL-6 $\left(\mathrm{IC}_{50}\right.$ $=8.14 \mu \mathrm{g} / \mathrm{ml})$ and TNF- $\alpha\left(\mathrm{IC}_{50}=1.49 \mu \mathrm{g} / \mathrm{ml}\right)$ in monocytes (Siriwatanametanon et al., 2010). The extract of $G$. pseudochina and its marker compounds of chlorogenic acid, caffeic acid, rutin, and $p$-coumaric acid also showed significant inhibition of IL-8 production in keratinocytes (Sukadeetad et al., 2018). G. segetum extract inhibited the release of TNF- $\alpha\left(\mathrm{IC}_{50}=\right.$ $16.20 \pm 3.94 \mu \mathrm{g} / \mathrm{ml})$ and $\mathrm{IL}-1 \beta\left(\mathrm{IC}_{50}=2.72 \pm 1.84 \mu \mathrm{g} / \mathrm{ml}\right)$ in macrophages. Among the isolated compounds of G. segetum, $4,5,4^{\prime}$-trihydroxychalcone was the most potent sample in inhibiting IL-1 $\beta \quad\left(\mathrm{IC}_{50}=6.69 \mu \mathrm{M}\right)$, and another isolated compound, rutin, demonstrated the strongest inhibition against $\mathrm{TNF}-\alpha$ release in macrophages $\left(\mathrm{IC}_{50}=16.96 \mu \mathrm{M}\right)$ (Yuandani et al., 2017). The anti-inflammatory effects of $G$. segetum and $G$. formosana have been demonstrated by the in vivo inhibition of TNF- $\alpha$ and IL-1, where the inhibitory effects of $500 \mathrm{mg} / \mathrm{kg}$ of $G$. segetum and $G$. formosana extracts were comparable to that of the nonsteroidal anti-inflammatory drug indomethacin (Seow et al., 2014; Ma et al., 2017). G. divaricata also suppressed TNF-a production in a diabetic mice model (Dong et al., 2019).

\section{Gynura Inhibits Prostaglandin $\mathrm{E}_{2}$ and Nitric Oxide Production}

Prostaglandin $\mathrm{E}_{2}\left(\mathrm{PGE}_{2}\right)$ is a bioactive lipid that physiologically mediates the regulation of immune responses, blood pressure, gastrointestinal integrity, and fertility. However, the sequential actions of cyclooxygenase-2 (COX-2) catalyze $\mathrm{PGE}_{2}$ synthesis. Consequently, dysregulated $\mathrm{PGE}_{2}$ production has been correlated to a variety of pathological conditions, such as chronic inflammation (Legler et al., 2010). Inhibition of COX-2 expression in mice model was exerted by $G$. procumbens essential oil and its active ingredients. Both G. procumbens essential oil and its active ingredients were showing similar COX-2 inhibitory effects as that of the positive control drug diclofenac diethylamine emulgel (Huang et al., 2019). The use of the G. bicolor extract inhibited $\mathrm{PGE}_{2}$ production and COX2 protein expression and activity in activated macrophages (Wu et al., 2013) and endothelial cells (Chao et al., 2015). PGE 2 production was also inhibited using the extract of $G$. pseudochina var. hispida with an $\mathrm{IC}_{50}$ value of $25.23 \mu \mathrm{g} / \mathrm{ml}$ (Siriwatanametanon et al., 2010).

Nitric oxide (NO) is a signaling molecule that physiologically exerts an anti-inflammatory effect and acts as a pro-inflammatory mediator in the overproduction of the enzyme iNOS (Sharma et al., 2007). G. procumbens extract had shown an inhibitory effect on NO production in macrophages (Liu M. et al., 2019; Ning et al., 2019; Chandradevan et al., 2020). This significant NO inhibitory effect by $250 \mu \mathrm{g} / \mathrm{ml} \mathrm{G}$ procumbens was comparable to the positive control of the non-selective NOS inhibitor $\mathrm{N} \omega$-nitro-l-arginine methyl ester hydrochloride (L-NAME) (Ning et al., 2019). G. procumbens fractions (petroleum ether, ethyl acetate, $n$-butanol, and water) at concentrations of $0.3-0.8 \mathrm{mg} / \mathrm{ml}$ also exerted $2-64 \%$ inhibition of NO production in macrophages. Among the fractions, the ethyl acetate fraction possessed the highest NO inhibitory effect (Liu M. et al., 2019). The protein expression of iNOS in macrophages was shown to be inhibited by the $G$. procumbens extract at a concentration of $250 \mu \mathrm{g} / \mathrm{ml}$ (Ning et al., 2019). Similarly, G. bicolor showed concentration dependent 


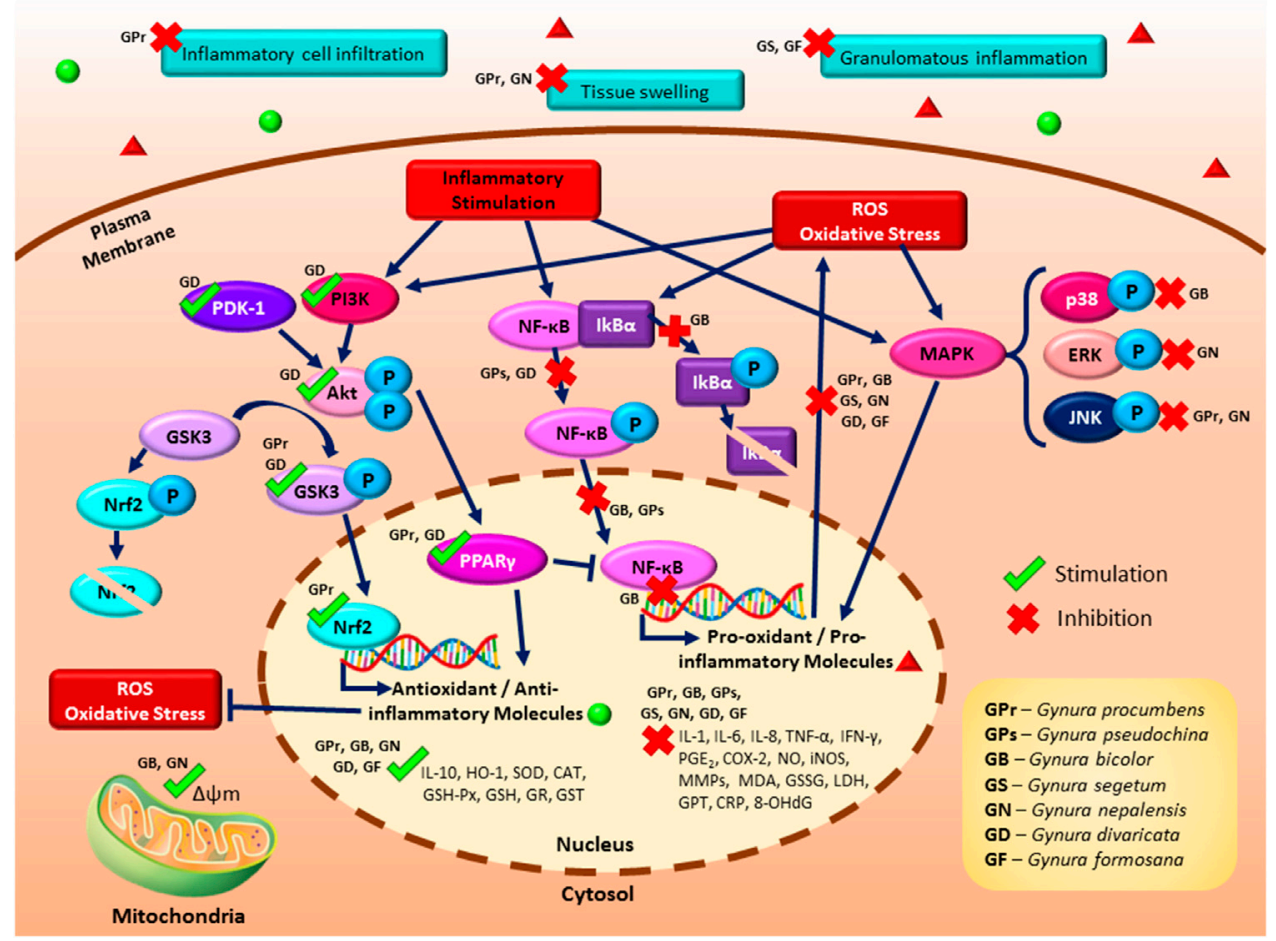

FIGURE 2 | A schematic illustrating the proposed signaling pathways of the antioxidant and anti-inflammatory effects of Gynura species. The antioxidant effects of Gynura are manifested by the inhibition of reactive oxygen species (ROS) production, suppression of lipid peroxidation, modulation of enzymatic antioxidant production or activities, and modulation of glutathione-related parameters. The reported anti-inflammatory effects of Gynura are modulation of inflammatory cytokines and molecule production as well as inhibition of cellular inflammation and inflammation in an animal model. The potential anti-inflammatory signaling pathways of Gynura include the induction of the phosphatidylinositol 3-kinase (PI3K)/protein kinase B (Akt), nuclear factor erythroid 2-related factor 2 (Nrf2), and peroxisome proliferator-activated receptor gamma (PPAR $\gamma$ ) signaling pathways as well as the inhibition of the nuclear factor kappa B (NF-kB), glycogen synthase kinase 3 (GSK3), and mitogen-activated protein kinase (MAPK) signaling pathways. Dark blue arrow lines indicate activation, dark blue perpendicular lines indicate inhibition, green tick indicates induction by Gynura species, and red cross indicates suppression by Gynura species.

suppression on iNOS protein expressions and NO production (30\% decrease with $120 \mu \mathrm{g} / \mathrm{ml}$ extract) in macrophages (Wu et al., 2013). G. segetum extract also inhibited NO production in macrophages, with $\mathrm{IC}_{50}=0.16 \pm 0.03 \mu \mathrm{g} / \mathrm{ml}$. Meanwhile, one of the compounds isolated from G. segetum extract, 8,8'-(ethene-1,2diyl)-dinaphtalene-1,4,5-triol, depicted the strongest NO inhibitory activity with an $\mathrm{IC}_{50}$ value of $0.15 \mu \mathrm{M}$ (Yuandani et al., 2017).

\section{Gynura Inhibits Cellular Inflammatory-Related Parameters}

Matrix metalloproteinases (MMPs) act as crucial regulatory enzymes in both pro- and anti-inflammatory pathways through the actions of cytokine or chemokine activation and antagonism (Manicone and McGuire, 2008). G. procumbens dose-dependently inhibited MMP-1 and MMP-9 expression in human dermal fibroblasts (HDFs), where the inhibition of the MMP-1 expression by the G. procumbens extract was even more effective than that of the positive control drug retinoic acid (Kim et al., 2011). Mitochondrial membrane potential $(\Delta \psi \mathrm{m})$, the energy provider to generate ATP, is produced when free energy is used to pump protons out of the mitochondrial matrix via oxidative phosphorylation. Under a stress condition, $\Delta \psi \mathrm{m}$ can be reduced and can possibly lead to mitochondrial dysfunction in inflammatory responses (Yue and Yao, 2016). In a concentration dependent manner, $\Delta \psi \mathrm{m}$ was increased, and LDH activity was inhibited by G. bicolor extract (Yang et al., 2019) and the isolated compound from the G. nepalensis, 3,5-dicaffeoylquinic acid ethyl ester (Yu et al., 2016). The induction of $\Delta \psi \mathrm{m}$ by the isolated compound of $G$. nepalensis was more effective than that of carbonyl cyanide $m$-chlorophenyl hydrazone, a chemical inhibitor of oxidative phosphorylation (Yu et al., 2016). G. formosana also suppressed plasma inflammatory biomarker (LDH, GPT, and CRP) activities in the treated group, where these inhibitory effects were comparable to indomethacin (Ma et al., 2017). The essential oil of G. procumbens and its active ingredient, limonene, showed an inhibitory effect on inflammatory cell infiltration (Huang et al., 2019).

\section{Gynura Inhibits Inflammation in Animal Models}

Tissue swelling (edema) is one of the cardinal signs of inflammation, where the increased fluid filtration is further enhanced by the arteriolar vasodilator action of the inflammatory mediators (Amelang et al., 1981). G. nepalensis extract diet (Rahman et al., 2018), topical administration of $G$. procumbens extract (Iskander et al., 2002), G. procumbens essential oil, and the active ingredients mixture from the $G$. procumbens essential oil (Huang et al., 2019) showed anti- 
TABLE 4 | List of studies on the anti-inflammatory effects of genus Gynura.

\begin{tabular}{|c|c|c|c|c|c|c|}
\hline Plant species & Part, Gynura form & Cell line/Animal study model & $\begin{array}{c}\text { Concentration/dose, control } \\
\text { groups }\end{array}$ & $\begin{array}{l}\text { Parameter measured and } \\
\text { technique used }\end{array}$ & Findings & Reference \\
\hline \multirow{12}{*}{$\begin{array}{l}\text { Gynura } \\
\text { procumbens (Lour.) } \\
\text { Merr. }\end{array}$} & Leaf & Human HaCaT keratinocytes & $100,500 \mu \mathrm{g} / \mathrm{ml}(24 \mathrm{~h})$ & $\begin{array}{l}\text { IL-6 and IL-8 production level using } \\
\text { ELISA }\end{array}$ & $\begin{array}{l}\text { By } 100 \text { and } 500 \mu \mathrm{g} / \mathrm{ml} \text { GP extract, IL- } \\
6 \text { and IL-8 were inhibited. }\end{array}$ & Kim et al. (2011) \\
\hline & Extract (Ethanol) & Human dermal fibroblasts (HDFs) & $1,10,20 \mu \mathrm{g} / \mathrm{ml}(48 \mathrm{~h})$ & $\begin{array}{l}\text { MMP-1 expression using western } \\
\text { blotting }\end{array}$ & $\begin{array}{l}\text { GP extract dose-dependently } \\
\text { inhibited MMP-1 and MMP-9 }\end{array}$ & \\
\hline & & $\begin{array}{l}\text { In vitro study stimulated by UV } \\
\text { irradiation }\end{array}$ & $\begin{array}{l}\text { Control groups: } \\
\text { Normal control } \\
\left.\text { Model control (UV } 40 \mathrm{~mJ} / \mathrm{cm}^{2}\right) \\
\text { Positive control }(10 \mu \mathrm{M} \text { Retinoic } \\
\text { acid) }\end{array}$ & $\begin{array}{l}\text { MMP-9 expression using } \\
\text { Zymography }\end{array}$ & expression in UV-B irradiated HDFs. & \\
\hline & $\begin{array}{l}\text { Leaf, stem } \\
\text { Essential oil and its active } \\
\text { ingredients }\end{array}$ & $\begin{array}{l}\text { RAW } 264.7 \text { macrophages } \\
\text { In vitro study stimulated by LPS }\end{array}$ & $\begin{array}{l}\text { GPEO: } 0.003,0.01,0.03 \mu \mathrm{g} / \mathrm{ml} \\
\text { Active ingredients: concentration not } \\
\text { stated } \\
\text { Treatment time not stated } \\
\text { Control groups: } \\
\text { Normal control } \\
\text { Model control (LPS) }\end{array}$ & $\begin{array}{l}\text { Inhibition on inflammatory cell } \\
\text { infiltrates using migration assay }\end{array}$ & $\begin{array}{l}\text { GPEO } \downarrow \downarrow \downarrow \text { LPS-induced cell } \\
\text { migration. Limonene, but not } \\
\alpha \text {-pinene, 3-carene, or their } \\
\text { components mixture, } \downarrow \downarrow \downarrow \text { cell } \\
\text { migration. }\end{array}$ & Huang et al. (2019) \\
\hline & $\begin{array}{l}\text { Extract (Ethanol) } \\
\text { Fractions (Petroleum ether, } \\
\text { ethyl acetate, n-butanol, } \\
\text { water) }\end{array}$ & In vitro study stimulated by LPS & $\begin{array}{l}0.4,0.6,0.8 \mathrm{mg} / \mathrm{ml} \\
24 \mathrm{~h} \text { incubation with LPS } \\
\text { Control groups: } \\
\text { Normal control } \\
\text { Model control }(1 \mu \mathrm{g} / \mathrm{ml} \text { LPS })\end{array}$ & $\begin{array}{l}\text { TNF- } \alpha \text { and IL-6 production level using } \\
\text { ELISA }\end{array}$ & $\begin{array}{l}\text { GP extract dose-dependently } \downarrow \text { IL-6. } \\
\text { GP extract at } 0.8 \mathrm{mg} / \mathrm{ml} \downarrow \text { TNF- } \alpha \text {. } \\
\text { Ethyl acetate fraction showed the } \\
\text { best inhibitory effect on IL-6 and TNF- } \\
\alpha .\end{array}$ & Liu M. et al. (2019) \\
\hline & Whole plant & RAW 264.7 macrophages & $\begin{array}{l}3.9,15.63,62.5 \text { and } 250 \\
\mu \mathrm{g} / \mathrm{ml}\end{array}$ & $\begin{array}{l}\text { Nitric oxide production level using } \\
\text { Griess assay }\end{array}$ & Pre-treatment of $250 \mu \mathrm{g} / \mathrm{ml} \mathrm{GP}:$ & Ning et al. (2019) \\
\hline & Extract (Ethanol) & In vitro study stimulated by LPS & $\begin{array}{l}1 \mathrm{~h} \text { pre-treatment } \\
\text { Control groups: } \\
\text { Normal control } \\
\text { Vehicle control }(0.1 \% \mathrm{DMSO}) \\
\text { Model control }(1 \mu \mathrm{g} / \mathrm{ml} \mathrm{LPS})\end{array}$ & $\begin{array}{l}\text { iNOS protein expression using } \\
\text { western blotting }\end{array}$ & $\begin{array}{l}\downarrow \text { NO production (dose dependent) } \\
\downarrow \text { iNOS protein expression. }\end{array}$ & \\
\hline & Leaf & $\begin{array}{l}\text { Mice normal liver cell line NCTC- } \\
1469\end{array}$ & 80 and $160 \mu \mathrm{g} / \mathrm{ml}(24 \mathrm{~h})$ & $\begin{array}{l}\text { Nrf2 and } p \text {-JNK protein expressions } \\
\text { using western blotting }\end{array}$ & $\begin{array}{l}80 \mu \mathrm{g} / \mathrm{ml} \mathrm{GP} \text { treatment } \uparrow \mathrm{Nr} 2 \text { protein } \\
\text { level, } \downarrow p \text {-JNK }\end{array}$ & Liu Y. et al. (2019) \\
\hline & Extract (Aqueous) & $\begin{array}{l}\text { In vitro study transfected with Ad- } \\
\text { shCFLAR }\end{array}$ & $\begin{array}{l}\text { Control groups: } \\
\text { Normal control (Ad-shCtrl) } \\
\text { Model control (pre-treated with Ad- } \\
\text { shCFLAR for } 24 \mathrm{~h} \text { ) }\end{array}$ & & $\begin{array}{l}160 \mu \mathrm{g} / \mathrm{ml} \mathrm{GP} \text { treatment } \uparrow \mathrm{Nrf2} \\
\text { protein levels, } \downarrow \downarrow p \text {-JNK }\end{array}$ & \\
\hline & Leaf & RAW 264.7 macrophages & $\begin{array}{l}15.63,31.25,62.5,125,250,500 \mu \mathrm{g} / \\
\mathrm{ml}\end{array}$ & $\begin{array}{l}\text { Nitric oxide production level using } \\
\text { Griess assay }\end{array}$ & $\begin{array}{l}\text { GP extract showed anti-inflammatory } \\
\text { activity by inhibiting NO production }\end{array}$ & $\begin{array}{l}\text { Chandradevan et al. } \\
\text { (2020) }\end{array}$ \\
\hline & Extract (Ethanol) & In vitro study stimulated by LPS & $\begin{array}{l}\text { Control groups: } \\
\text { Normal control } \\
\text { Model control (IFN- } \gamma+\text { LPS) }\end{array}$ & & & \\
\hline & $\begin{array}{l}\text { Aerial part } \\
\text { Extract (Ethanol) }\end{array}$ & $\begin{array}{l}\text { Balb/c white mice (5 mice/group) } \\
\text { In vivo study using croton oil- } \\
\text { induced ear inflammation mice } \\
\text { model }\end{array}$ & $\begin{array}{l}0.75 \mathrm{mg} / 20 \mu \mathrm{l} \\
\text { Topical application } \\
30 \text { min pre-treatment, ear thickness } \\
\text { was measured at } 24 \mathrm{~h} \\
\text { Control groups: } \\
\text { Model control ( } 20 \mu \mathrm{l} \text { acetone at left } \\
\text { ear }+0.1 \mathrm{mg} / 20 \mu \mathrm{l} / \mathrm{ear} \text { croton oil) } \\
\text { Standard anti-inflammatory agent } \\
\text { (hydrocortisone) }\end{array}$ & $\begin{array}{l}\text { Ear thickness (anti-inflammatory } \\
\text { activity) }\end{array}$ & $\begin{array}{l}\text { Original organic crude extract } \downarrow \downarrow \downarrow \\
\text { croton oil-induced ear inflammation. }\end{array}$ & Iskander et al. (2002) \\
\hline
\end{tabular}


TABLE 4 | (Continued) List of studies on the anti-inflammatory effects of genus Gynura.

\begin{tabular}{|c|c|c|c|c|c|c|}
\hline Plant species & Part, Gynura form & Cell line/Animal study model & $\begin{array}{c}\text { Concentration/dose, control } \\
\text { groups }\end{array}$ & $\begin{array}{l}\text { Parameter measured and } \\
\text { technique used }\end{array}$ & Findings & Reference \\
\hline & Leaf & Male BALB/c mice (5 mice/group) & $50 \mathrm{mg} / \mathrm{kg}$ body weight & $\begin{array}{l}\text { GSK3 } \beta \text { phosphorylation using } \\
\text { western blotting }\end{array}$ & $\begin{array}{l}\text { GP } \uparrow \text { phosphorylation of liver GSK3 } \beta \\
\text { (Ser9) compared with non-treated } \\
\text { control }\end{array}$ & Wong et al. (2015) \\
\hline & \multirow[t]{3}{*}{ Extract (Ethanol) } & \multirow[t]{3}{*}{$\begin{array}{l}\text { In vivo study using parasite- } \\
\text { infected mice model }\end{array}$} & Intraperitoneally treated & \multirow[t]{3}{*}{$\begin{array}{l}\text { TNF-a, IFN- } \gamma \text { and IL-10 production } \\
\text { level using ELISA }\end{array}$} & \multirow{3}{*}{$\begin{array}{l}\downarrow \text { TNF- } \alpha \text { and IFN- } \gamma \text { levels in liver and } \\
\text { serum by administration of GP } \\
\uparrow \text { IL-10 level in serum. }\end{array}$} & \\
\hline & & & 1 day pre-infection & & & \\
\hline & & & $\begin{array}{l}\text { Control groups: } \\
\text { Normal control } \\
\text { Model control (B. pseudomallei } \\
\text { infection) }\end{array}$ & & & \\
\hline & Leaf, stem & $\begin{array}{l}50 \text { male Kunming mice (5 mice/ } \\
\text { group) }\end{array}$ & GPEO: $0.433,0.865,1.73 \mathrm{mg} / \mathrm{ml}$ & $\begin{array}{l}\text { Ear thickness by xylene-induced ear } \\
\text { edema }\end{array}$ & \multirow{2}{*}{$\begin{array}{l}\text { hind paw edema at all doses } \\
\text { throughout experiment. Treatment } \\
\text { with active ingredients mixture } \downarrow \text { ear } \\
\text { and hind paw edema. }\end{array}$} & \multirow[t]{5}{*}{ Huang et al. (2019) } \\
\hline & \multirow[t]{4}{*}{$\begin{array}{l}\text { Essential oil and its active } \\
\text { ingredients }\end{array}$} & \multirow[t]{4}{*}{$\begin{array}{l}\text { In vivo study using xylene and } \\
\text { formalin-induced inflammation } \\
\text { mice model }\end{array}$} & $\begin{array}{l}\text { Active ingredients: } \boldsymbol{\alpha} \text {-pinene } \\
(0.174 \mathrm{mg} / \mathrm{ml}), 3 \text {-carene }(0.153 \mathrm{mg} / \\
\mathrm{ml}) \text {, limonene }(0.036 \mathrm{mg} / \mathrm{ml}) \text { and } \\
\text { mixture of all } 3 \text { ingredients }\end{array}$ & $\begin{array}{l}\text { Hind paw edema using micrometre } \\
\text { and histological examination }\end{array}$ & & \\
\hline & & & Topical administration & \multirow[t]{3}{*}{$\begin{array}{l}\text { COX-2 expression using } \\
\text { immunohistochemical staining and } \\
\text { multispectral imaging analysis }\end{array}$} & \multirow{3}{*}{$\begin{array}{l}\downarrow \text { COX-2 expression by } 0.865 \mathrm{mg} / \mathrm{ml} \\
\text { GPEO and all } 3 \text { individual and mixture } \\
\text { of active ingredients. } \\
\downarrow \downarrow \text { COX-2 expression by } 1.73 \mathrm{mg} / \mathrm{ml} \\
\text { GPEO. }\end{array}$} & \\
\hline & & & $\begin{array}{l}\text { Control groups: } \\
\text { Untreated control }\end{array}$ & & & \\
\hline & & & $\begin{array}{l}\text { Model control (topical application of } \\
\text { sesame oil + } 20 \boldsymbol{\mu l} \text { xylene, } \\
\text { intradermal injection of } 20 \boldsymbol{\mu l} 4 \% \\
\text { formalin) } \\
\text { Positive control (diclofenac } \\
\text { diethylamine emulgel (DDE)) }\end{array}$ & & & \\
\hline & Leaf & $\begin{array}{l}32 \text { Male C57BL/6J mice (8 mice/ } \\
\text { group) }\end{array}$ & 500 and $1,000 \mathrm{mg} / \mathrm{kg}$ body weight & $\begin{array}{l}\text { Nrf2 and } p \text {-JNK protein expressions } \\
\text { using western blotting }\end{array}$ & $\begin{array}{l}500 \mathrm{mg} / \mathrm{kg} \text { GP treatment } \uparrow \mathrm{Nrf} 2 \\
\text { protein level, no obvious effect on } p \text { - }\end{array}$ & Liu Y. et al. (2019) \\
\hline & \multirow[t]{4}{*}{ Extract (Aqueous) } & \multirow[t]{4}{*}{$\begin{array}{l}\text { In vivo study using non-alcoholic } \\
\text { steatohepatitis (NASH) mic }\end{array}$} & $\begin{array}{l}\text { Oral route } \\
\text { Daily for } 6 \text { weeks }\end{array}$ & $\begin{array}{l}\text { mRNA expression of PPARy using } \\
\text { RT-qPCR }\end{array}$ & \multirow{4}{*}{$\begin{array}{l}\text { protein level, no obvious effect on } p \text { - } \\
\text { JNK, } \uparrow \text { PPAR } y \text { mRNA expression. } \\
1000 \mathrm{mg} / \mathrm{kg} \text { GP treatment } \uparrow \text { protein } \\
\text { levels of hepatic Nrf2, } \downarrow \text { protein level } \\
\text { of hepatic } p \text {-JNK, } \uparrow \uparrow \text { PPAR } y \text { mRNA } \\
\text { expression. }\end{array}$} & \\
\hline & & & Control groups: & & & \\
\hline & & & & & & \\
\hline & & & $\begin{array}{l}\text { Model control (methionine- and } \\
\text { choline-deficient (MCD) diet) }\end{array}$ & & & \\
\hline \multirow[t]{9}{*}{$\begin{array}{l}\text { Gynura bicolor } \\
\text { (Roxb. ex Willd.) DC }\end{array}$} & Leaf & RAW 264.7 macrophages & $15,30,60$, or $120 \mu \mathrm{g} / \mathrm{ml}$ & $\begin{array}{l}\text { Nitric oxide production level using } \\
\text { Griess assay }\end{array}$ & $\begin{array}{l}\downarrow \text { NO production (30\% decrease with } \\
120 \mu \mathrm{gg} / \mathrm{ml} \mathrm{GB} \text { ) }\end{array}$ & Wu et al. (2013) \\
\hline & \multirow[t]{8}{*}{ Extract (Ether) } & \multirow[t]{8}{*}{ In vitro study stimulated by LPS } & $3 \mathrm{~h}$ pre-treatment & $\begin{array}{l}\text { PGE2 production using competitive } \\
\text { enzyme immunoassay (EIA) kit }\end{array}$ & $\downarrow$ PGE2 production (dose dependent) & \\
\hline & & & Control groups: & $\begin{array}{l}\text { iNOS, COX-2, phosphorylated-IkBa } \\
\text { and p65 protein expression using } \\
\text { immunoblot analysis }\end{array}$ & $\begin{array}{l}\downarrow \text { iNOS and COX-2 protein } \\
\text { expressions (concentration } \\
\text { dependent) }\end{array}$ & \\
\hline & & & $\begin{array}{l}\text { Vehicle control group (0.1\% (v/v) } \\
\text { methanol) }\end{array}$ & $\begin{array}{l}\text { NF-kB DNA-binding activity by } \\
\text { Nuclear protein preparation and }\end{array}$ & $\begin{array}{l}\downarrow p \text { - } \mathbf{k B a} \text { protein (47\% decrease with } \\
120 \mu \mathrm{\mu g} / \mathrm{ml} \mathrm{GB})\end{array}$ & \\
\hline & & & Model control (1 $\mu \mathrm{g} / \mathrm{ml}$ LPS) & electrophoretic mobility shift assay & $\downarrow$ nucleic p65 protein levels (3-41\% & \\
\hline & & & & (EMSA & decrease with 30, 60, $120 \mu \mathrm{gg} / \mathrm{ml} \mathrm{GB}$ ) & \\
\hline & & & & & $\begin{array}{l}\downarrow \text { translocation of NF-kB from the } \\
\text { cytosol to nuclei }\end{array}$ & \\
\hline & & & & & $\downarrow$ DNA-binding activity of NF-kB & \\
\hline & & & & & $\begin{array}{l}\text { nuclear protein by } 30,60,120 \mu \mathrm{g} / \mathrm{ml} \\
\text { GB }\end{array}$ & \\
\hline
\end{tabular}


TABLE 4 | (Continued) List of studies on the anti-inflammatory effects of genus Gynura.

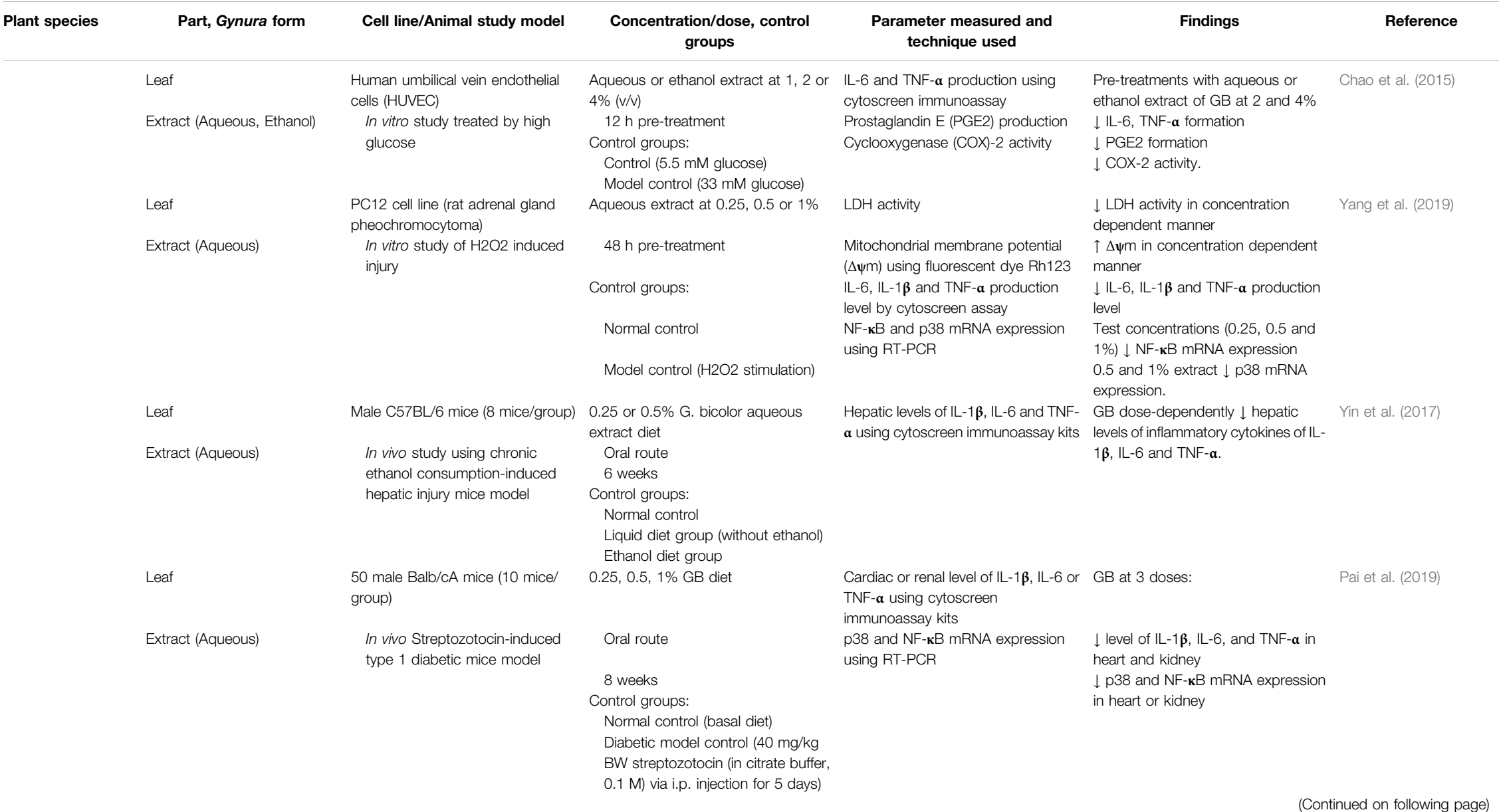


TABLE 4 | (Continued) List of studies on the anti-inflammatory effects of genus Gynura.

\begin{tabular}{|c|c|c|c|c|c|c|}
\hline Plant species & Part, Gynura form & Cell line/Animal study model & $\begin{array}{c}\text { Concentration/dose, control } \\
\text { groups }\end{array}$ & $\begin{array}{l}\text { Parameter measured and } \\
\text { technique used }\end{array}$ & Findings & Reference \\
\hline \multirow[t]{9}{*}{$\begin{array}{l}\text { Gynura } \\
\text { pseudochina (L.) } \\
\text { DC. }\end{array}$} & $\begin{array}{l}\text { Leaf } \\
\text { Extract (Methanol, Ethyl } \\
\text { acetate, Petroleum ether) }\end{array}$ & $\begin{array}{l}\text { HeLa cells } \\
\text { In vitro study stimulated by PMA } \\
\text { Monocytes from healthy human } \\
\text { donors } \\
\text { In vitro study stimulated by LPS }\end{array}$ & $\begin{array}{l}0.2-200 \mu \mathrm{g} / \mathrm{ml} \\
1,10 \text { and } 50 \mu \mathrm{g} / \mathrm{ml} \\
24 \mathrm{~h} \\
\text { Control groups: } \\
\text { Positive control (ethanol) } \\
\text { Negative control (Unstimulated cells) } \\
\text { Reference group (Parthenolide, } \\
\text { hydrocortisone) }\end{array}$ & $\begin{array}{l}\text { IL-6/luciferase assay (NF-kB assay) } \\
\text { IL-6, IL-1, TNF- } \boldsymbol{\alpha} \text { and PGE2 } \\
\text { production level using ELISA and EIA }\end{array}$ & $\begin{array}{l}\text { Gynura pseudochina var. hispida } \\
\text { (MeOH) showed the strongest NF-kB } \\
\text { inhibitory effects, as well as inhibition } \\
\text { on release of IL-1, IL-6, TNF- } \boldsymbol{\alpha} \text { and } \\
\text { PGE2. }\end{array}$ & $\begin{array}{l}\text { Siriwatanametanon et al. } \\
\text { (2010) }\end{array}$ \\
\hline & Leaf & HeLa cells & $\begin{array}{l}\text { Non-toxic concentrations (using the } \\
\text { MTT assay) }\end{array}$ & IL-6/luciferase assay (NF-kB assay) & $\begin{array}{l}\text { Quercetin 3-rutinoside showed the } \\
\text { highest NF-kB inhibitory effect. }\end{array}$ & $\begin{array}{l}\text { Siriwatanametanon and } \\
\text { Heinrich (2011) }\end{array}$ \\
\hline & \multirow[t]{3}{*}{$\begin{array}{l}\text { Isolated compound from } \\
\text { methanol extract }\end{array}$} & \multirow[t]{3}{*}{ In vitro study stimulated by PMA } & $\begin{array}{l}\text { Control groups: } \\
\text { Positive control (ethanol) }\end{array}$ & & $\begin{array}{l}\text { NF-kB inhibitory activities IC50: } \\
\text { Quercetin 3-rutinoside: } 24.1 \pm \\
0.1 \mu \mathrm{\mu g} / \mathrm{ml}\end{array}$ & \\
\hline & & & Negative control (Unstimulated cells) & & $\begin{array}{l}\text { 3,5-di-caffeoylquinic acid: } 42.8 \pm \\
0.2 \mu \mathrm{g} / \mathrm{ml}\end{array}$ & \\
\hline & & & Reference group (Parthenolide) & & $\begin{array}{l}\text { 4,5-di-caffeoylquinic acid: } 49.1 \pm \\
0.1 \mu \mathrm{g} / \mathrm{ml} \\
\text { 5-mono-caffeoylquinic acid: } 83.0 \pm \\
0.1 \mu \mathrm{g} / \mathrm{ml}\end{array}$ & \\
\hline & Leaf & Human HaCaT keratinocytes & Extract: 375 and $750 \mu \mathrm{g} / \mathrm{ml}$ & IL-8 production level using ELISA & $\begin{array}{l}\text { Extract at both tested concentrations } \\
\text { and some concentration of each } \\
\text { marker compounds: }\end{array}$ & Sukadeetad et al. (2018) \\
\hline & $\begin{array}{l}\text { Extract (Ethanol), marker } \\
\text { compounds }\end{array}$ & In vitro study stimulated by TNF-a & $\begin{array}{l}\text { Chlorogenic acid: } 140 \text { and } 280 \mu \mathrm{\mu g} / \mathrm{ml} \\
\text { Caffeic acid: } 30 \text { and } 60 \mu \mathrm{g} / \mathrm{ml} \\
\text { Rutin: } 750 \text { and } 1,500 \mu \mathrm{g} / \mathrm{ml} \\
\text { p-coumaric acid: } 1,400 \text { and 2,800 } \mu \mathrm{g} / \\
\mathrm{ml} \\
24 \mathrm{~h} \\
\text { Control groups: } \\
\text { Normal control } \\
\text { Model control ( } 50 \mathrm{ng} / \mathrm{ml} \text { TNF- } \boldsymbol{\alpha} \text { with/ } \\
\text { without } 0.7 \% \text { DMSO) } \\
\text { Positive control ( } 50 \mu \mathrm{g} / \mathrm{ml} \text { curcumin) }\end{array}$ & $\begin{array}{l}\text { RelA and RelB localization by } \\
\text { immunofluorescence assay }\end{array}$ & $\begin{array}{l}\downarrow \mathrm{LL}-8 \text { production level } \\
\downarrow \text { translocation of RelB S573 into } \\
\text { nucleus }\end{array}$ & \\
\hline & Leaf & $\begin{array}{l}25 \text { patients with mild to moderate } \\
\text { plaque psoriasis }\end{array}$ & Mixture of extract and vehicle (1:10) & $\begin{array}{l}\text { Phosphorylation of NF-kB p65 using } \\
\text { immunohistochemistry (skin sections }\end{array}$ & $\begin{array}{l}\text { Immunohistochemical staining } \\
\text { revealed diminution of }\end{array}$ & Rerknimitr et al. (2016) \\
\hline & Ointment from ethanol extract & Randomized controlled study & $\begin{array}{l}\text { Twice daily for } 4 \text { weeks } \\
\text { Control: } 0.1 \% \text { triamcinolone cream }\end{array}$ & from two patients) & $\begin{array}{l}\text { phosphorylated NF-kB p65 in the } \\
\text { lesions treated with the GP ointment. }\end{array}$ & tinued on following page) \\
\hline
\end{tabular}


TABLE 4 | (Continued) List of studies on the anti-inflammatory effects of genus Gynura.

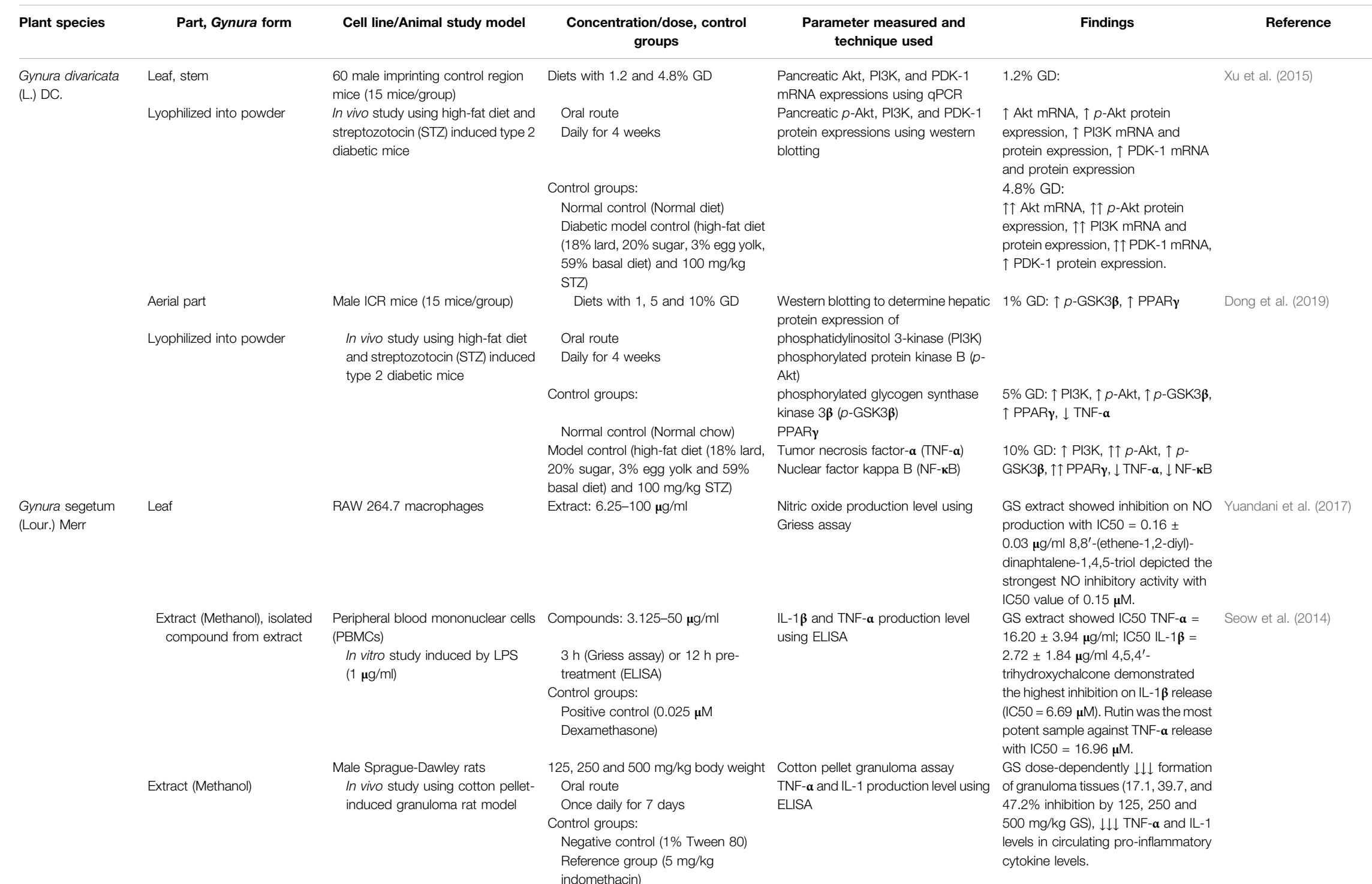


TABLE 4 | (Continued) List of studies on the anti-inflammatory effects of genus Gynura.

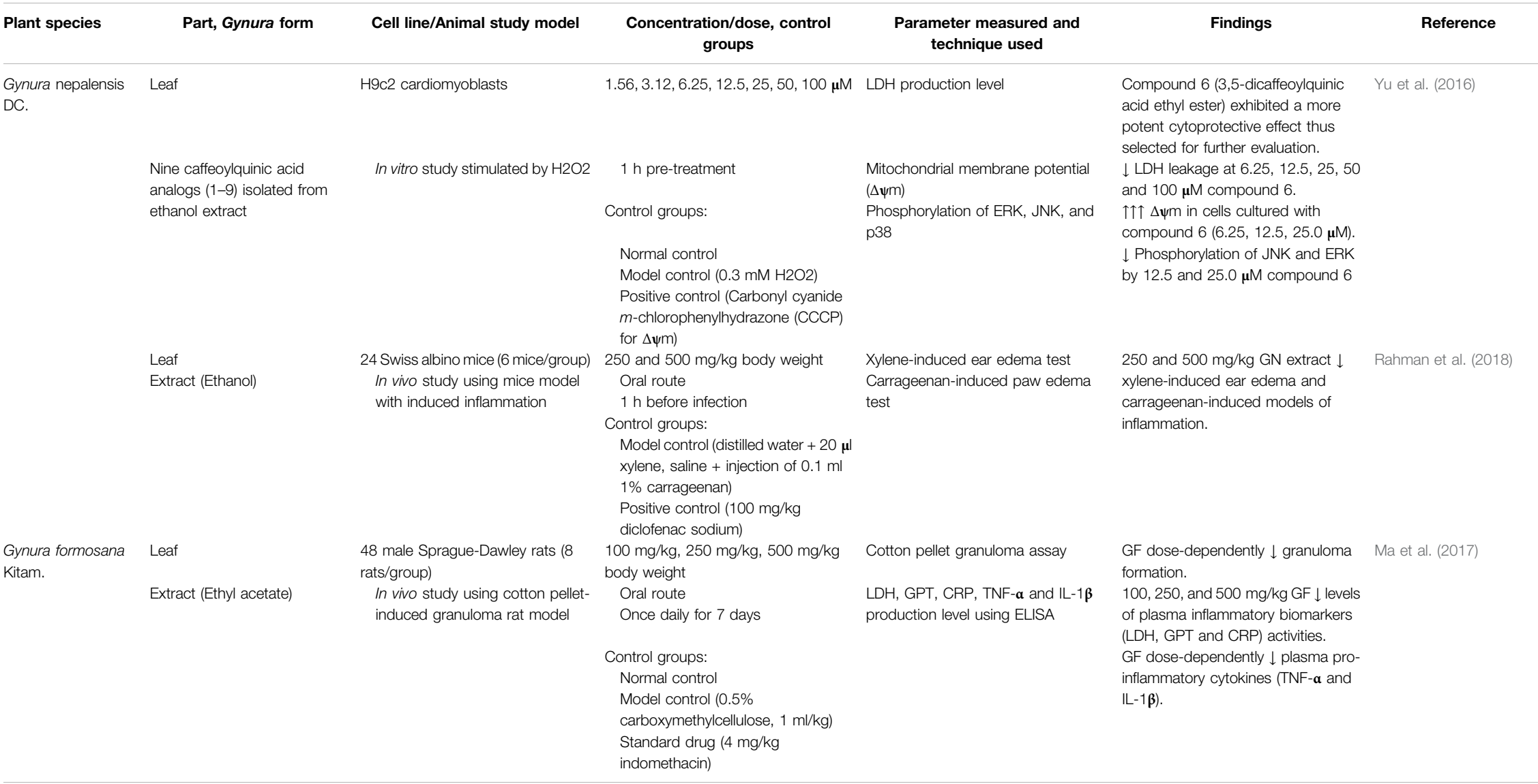

$\uparrow$ indicates significantly induce $(\mathrm{p}<0.05)$, $\uparrow$ indicates significantly induce $(\mathrm{p}<0.01)$, $\uparrow \uparrow \uparrow$ indicates significantly induce $(\mathrm{p}<0.001), \downarrow$ indicates significantly inhibit $(\mathrm{p}<0.05), \downarrow \downarrow \downarrow$ indicates significantly inhibit $(\mathrm{p}<0.01)$, and $\downarrow \downarrow \downarrow$ indicates significantly inhibit $(\mathrm{p}<0.001)$ 
inflammatory effects by significantly reducing ear thickness and paw edema in inflammatory mice model. Granulomatous inflammation is a special variety of chronic inflammation in which cells of the mononuclear phagocyte system are aggregated into well-demarcated focal lesions called granulomas (Williams and Williams, 1983). The anti-inflammatory effect of G. segetum and $G$. formosana had been demonstrated by significant inhibition of granuloma tissue formation in a rat model (Seow et al., 2014; Ma et al., 2017).

\section{Anti-inflammatory Signaling Pathways of Gynura}

Mitogen-activated protein kinases (MAPK) consist of three signaling pathways: ERK, JNK, and p38 MAPK, which mediate fundamental cellular processes by regulating immunomodulatory cytokine expression (Kaminska, 2005). G. bicolor extract possessed an anti-inflammatory effect by inhibiting p38 mRNA expression both in vitro (Yang et al., 2019) and in vivo (Pai et al., 2019). The isolated compound from $G$. nepalensis extract, 3,5-dicaffeoylquinic acid ethyl ester, exhibited inhibitory effects on the phosphorylation of JNK and ERK in cardiomyoblasts (Yu et al., 2016). Similarly, G. procumbens treatment also inhibited the protein expression of p-JNK in murine hepatocytes and in a mice model (Liu Y. et al., 2019). NF-кB represents a family of inducible transcription factors that activate the transcription of various pro-inflammatory genes (Liu et al., 2017). The NF-кB signaling pathway had been proposed as a potential mechanism of the anti-inflammatory effect of $G$. bicolor through the inhibition of the $\mathrm{p}-\mathrm{I}_{\kappa} \mathrm{B} \alpha$ protein, nucleic $\mathrm{p} 65$ protein levels, translocation of $\mathrm{NF}-\mathrm{k}$ from the cytosol to the nuclei, and DNA-binding activity of the NF- $\mathrm{BB}$ nuclear protein (Wu et al., 2013). To complement the study by Wu et al. (2013), two studies demonstrated that the G. bicolor aqueous extract was able to inhibit NF-кB mRNA expression in both in vitro (Yang et al., 2019) and in vivo studies (Pai et al., 2019). Meanwhile, $G$. pseudochina var. hispida extract caused the inhibition of $\mathrm{NF}_{-\kappa} \mathrm{B}$ activation with $\mathrm{IC}_{50}=41.96 \mu \mathrm{g} / \mathrm{ml}$ in HeLa cells (Siriwatanametanon et al., 2010). Successively, quercetin 3rutinoside, which was one of the isolated compounds from $G$. pseudochina var. hispida extract, showed a strong NF- $\mathrm{kB}$ inhibitory effect with $\mathrm{IC}_{50}=24.1 \pm 0.1 \mu \mathrm{g} / \mathrm{ml}$ (Siriwatanametanon and Heinrich, 2011). G. pseudochina extract and its marker compounds of chlorogenic acid, caffeic acid, rutin, and $p$ coumaric acid also inhibited RelB S573 (protein transcription factors of $\mathrm{NF}_{\kappa} \mathrm{B}$ family) translocation into the nucleus of keratinocytes (Sukadeetad et al., 2018). Moreover, G. divaricata lyophilized powder at $10 \%$ of diet inhibited the NF-кB signaling pathway (Dong et al., 2019). In a randomized controlled study, NF$\kappa \mathrm{B}$ phosphorylation in the lesions treated with G. pseudochina ointment was inhibited (Rerknimitr et al., 2016).

Upon cellular induction, phosphoinositide 3-kinases (PI3K) generate lipid products that recruit cytosolic protein kinase $\mathrm{B}$ (Akt) to cellular membranes for Akt activation. Phosphoinositide-dependent kinase-1 (PDK-1) is a serine/ threonine kinase that facilitates Akt phosphorylation. The activated Akt detaches from the plasma membrane and translocates through the cytosol to the nucleus for further downstream reactions (Vanhaesebroeck and Alessi, 2000). G. divaricata lyophilized powder in the diet of a diabetic mice model significantly induced the mRNA and protein expression of Akt and PI3K (Xu et al., 2015; Dong et al., 2019). Similarly, a mice model with $G$. divaricata lyophilized powder in its diet also exhibited significant induction of the mRNA and protein expression of PDK-1 (Xu et al., 2015). By Akt-mediated phosphorylation, the PI3K/Akt signaling pathway was able to inactivate GSK3 (Salazar et al., 2006), where GSK3 has been determined as one of the mechanisms that phosphorylate and degrade Nrf2. In turn, Nrf2, a transcription factor, will be upregulated and will ultimately lead to the production of downstream cytoprotective protein expression (Cuadrado et al., 2018). GSK3 acts as the central regulator of the inflammatory response to bacterial infections and other insults. Hence, inactivation of GSK3 by the phosphorylation of GSK $3 \beta$ has been proposed as a potential therapeutic target in the control of bacterial-driven inflammatory diseases (Wang et al., 2014). The anti-inflammatory effect of $G$. procumbens was correlated to the increased phosphorylation of liver GSK3 $\beta$ (Ser9), which inhibited the activities of GSK3 (Wong et al., 2015). G. divaricata lyophilized powder diet increased GSK3 $\beta$ phosphorylation in a diabetic mice model (Dong et al., 2019). G. procumbens treatment showed anti-inflammatory potential through the induction of the Nrf2 protein level in murine hepatocytes and in a mice model (Liu Y. et al., 2019). Akt activation is also essential for the transcriptional activation of peroxisome proliferator-activated receptor- $\gamma\left(\operatorname{PPAR}_{\gamma}\right)$ (Kim et al., 2010), a nuclear receptor that inhibits the expression of inflammatory cytokines and directs the differentiation of immune cells toward anti-inflammatory phenotypes (Tyagi et al., 2011). G. procumbens extract in diet significantly induced in vivo PPAR $\gamma$ mRNA expression (Liu Y. et al., 2019), and $G$. divaricata lyophilized powder diet increased in vivo hepatic protein expression of PPAR $\gamma$ (Dong et al., 2019).

\section{Interplay Between Physiological, Biochemical and Immunological Aspects}

The antioxidant and anti-inflammatory effects of Gynura species are corroborated to each other. As aforementioned, inflammation and oxidative stress are highly interdependent pathophysiological events. Hence the antioxidant effects of Gynura species has supported anti-inflammatory effects by these plants. In general, Gynura species depict anti-inflammatory effects by inhibiting inflammatory signaling pathways, cellular pathogenicity, inflammatory biomolecules secretion, and clinical manifestation of inflammatory diseases. Granulomatous inflammation is the end result of a prolonged complex interplay among causal agents, mononuclear phagocytes activity, circulating immune complexes, and a vast array of biological mediators (Zumla and James, 1996). Inflammatory cell infiltration is the critical process in granuloma formation while tissue swelling is one of the possible associated clinical manifestation. The pathogenesis of granulomatous inflammation is manipulated by the vital player of macrophages along with secretion of cytokines and chemokines by immune cells through stimulation of inflammatory signaling pathways (Facco et al., 2007). Not only granulomatous inflammation, the signaling pathways, biomolecules and cellular responses also contribute 
TABLE 5 | Phytochemicals of Gynura species.

Species

Gynura procumbens (Lour.) Merr.
Identified phytochemicals

15,16-Dihydroxy-9Z, 12Z-octadecadienoic acid (PubChem CID: 16061068)

3,4-Dicaffeoylquinic acid (Isochlorogenic acid B) (PubChem CID: 5281780)

3,5-Dicaffeoylquinic acid (Isochlorogenic acid A) (PubChem CID:

6474310)

3,5-O-Dicaffeoylquinic acid (PubChem CID: 13604688)

3-O-Methyl gallic acid sulfate (PubChem CID: not found)

3-Carene (PubChem CID: 26049)

4,5-Dicaffeoylquinic acid (Isochlorogenic acids C) (PubChem CID:

6474309)

4-O-Methyl gallic acid sulfate (PubChem CID: not found)

5-O-(E)-Caffeoyl-galactaric acid (PubChem CID: not found)

Apigenin (PubChem CID: 5280443)

Caffeic acid (PubChem CID: 689043)

Chlorogenic acid (PubChem CID: 1794427)

Choline (PubChem CID: 305)

Citric acid (PubChem CID: 311)

Cynarine (PubChem CID: 5281769)

Dicaffeoylquinic acids (PubChem CID: 6474310)

Eriocitrin (PubChem CID: 83489)

Ferulic acid (PubChem CID: 445858)

Feruloylquinic acid (PubChem CID: 10133609)

Gallic acid (PubChem CID: 370)

Genkwanin isomer (PubChem CID: 5281617)

Isobioquercetin (PubChem CID: not found)

Kaempferol (PubChem CID: 5280863)

Kaempferol 3-O-glucoside (Astragalin) (PubChem CID: 5282102)

Kaempferol 3-O-rhamnosyl-(1 $\rightarrow 6)$-glucoside (PubChem CID: not

found)

Kaempferol-3-O-rutinoside (Nicotiflorin) (PubChem CID:

5318767)

Limonene (PubChem CID: 22311)

Malic acid (PubChem CID: 525)

Myricetin (PubChem CID: 5281672)

Neochlorogenic acid (PubChem CID: 5280633)

Oxooctadecanoic acid (PubChem CID: 439332)

p-Coumaric acid (PubChem CID: 637542)

p-Coumaroylquinic acid (PubChem CID: 6441280)

p-Hydroxybenzoic acid (PubChem CID: 135)

Phenylalanine (PubChem CID: 6140)

Protocatechuic acid (PubChem CID: 72)

Quercetin (PubChem CID: 5280343)

Quercetin 3-O-rhamnosyl-(1 $\rightarrow 2)$-galactoside (PubChem CID: 44259099)

Quercetin 3-O-rhamnosyl-(1 $\rightarrow 6)$-glucoside (PubChem CID: not

found)

Quercetin 3-O-rutinoside (Rutin) (PubChem CID: 5280805)

Sinapic acid (PubChem CID: 637775)

Syringic acid (PubChem CID: 10742)

Trimethyl gallic acid glucuronide (PubChem CID: not found)

Vanillic acid (PubChem CID: 8468)

a-Pinene (PubChem CID: 6654)

\section{References}

Akowuah et al. (2002), Rosidah et al. (2008), Kim et al. (2011), Kaewseejan and Siriamornpun (2015), Murugesu et al. (2017), Li et al. (2018), Murugaiyah et al. (2018), Nazri et al. (2019), Huang et al. (2019), Liu M. et al. (2019), Liu Y. et al. (2019), Chandradevan et al. (2020) 
TABLE 5 | (Continued) Phytochemicals of Gynura species.

Species

Identified phytochemicals

References

Gynura bicolor (Roxb. ex Willd.) DC. 3,5-Di-O-caffeoylquinic acid (Isochlorogenic acid A) (PubChem CID: 6474310)

3-O-Feruloylquinic acid (PubChem CID: 9799386)

3-O-p-Coumaroylquinic acid (PubChem CID: 9945785)

4,5-Di-O-Caffeoylquinic acid (Isochlorogenic acid C) (PubChem

CID: 6474309)

5-O-Caffeoylquinic acid (Neochlorogenic Acid) (PubChem CID:

5280633)

5-O-p-Coumaroylquinic acid (PubChem CID: 9945785)

Anthocyanin (PubChem CID: 145858)

Caffeoyl glucose (PubChem CID: 129715972)

Citric acid (PubChem CID: 311)

Dihydro-phellopterin (PubChem CID: not found)

Gallic acid (PubChem CID: 370)

Geniposide (PubChem CID: 107848)

Guanosine (PubChem CID: 135398635)

Isobavachalcone (PubChem CID: 5281255)

Kaempferol-3-O-caffeoylate (PubChem CID: not found)

Kaempferol-3-O-glucoside (Astragalin) (PubChem CID: 5282102)

Malic acid (PubChem CID: 525)

Phenylalanine (PubChem CID: 6140)

Protocatechuate-O-glucoside (PubChem CID: not found)

Quercetin (PubChem CID: 5280343)

Quercetin-3-acetylhexose (PubChem CID: not found)

Quercetin-3-O-galactoside (PubChem CID: 5281643)

Quercetin-3-O-rutinoside (Rutin) (PubChem CID: 5280805)

Tryptophan (PubChem CID: 6305)

Uridine (PubChem CID: 6029)

$\boldsymbol{\beta}$-Carotene (PubChem CID: 5280489)

Gynura pseudochina (L.) DC.
Lu et al. (2012), Wu et al. (2015), Qiu X. L. et al. (2018)
(+)-Tephropurpurin (PubChem CID: 10047971)

1-(9Z-octadecenoyl)-sn-glycero-2,3-cyclic phosphate (PubChem CID: 52922109)

1,3,8-Trihydroxy-4-methyl-2,7-diprenylxanthone (PubChem CID: 67261902)

2-(2,4-Dihydroxyphenyl)-5-hydroxy-8-methyl-8-(4-methyl-3-

penten-1-yl)-2,3-dihydro-4H,8H-pyrano[2,3-f]chromen-4-one

(PubChem CID: not found)

3,4-Dihydroxycinnamoyl-(Z)-2-(3,4-Dihydroxyphenyl) Ethanol

(PubChem CID: 14353342)

3,5-Dicaffeoyl quinic acid (Isochlorogenic acid A) (PubChem CID: 6474310)

3-O-Caffeoyl-1-O-methylquinic acid (PubChem CID: 131752768)

4,5-Dicaffeoyl quinic acid (PubChem CID: 13887346)

5-Hydroxy-2' -methoxy-6,7-methylenedioxyisoflavone (PubChem CID: 5491929)

5-Caffeoyl quinic acid (Chlorogenic acid) (PubChem CID:

1794427)

Caffeic acid (PubChem CID: 689043)

Isochlorogenic acid B (PubChem CID: 5281780)

Isochlorogenic acid C (PubChem CID: 6474309)

Kaempferol rutinoside (Nicotiflorin) (PubChem CID: 5318767)

Quercetin (PubChem CID: 5280343)

Quercetin 3-rutinoside (Rutin) (PubChem CID: 5280805)

Stigmasterol (PubChem CID: 5280794)

$\boldsymbol{\beta}$-Sitosterol (PubChem CID: 222284)
Siriwatanametanon and Heinrich (2011), Ferlinahayati et al. (2017), Sukadeetad et al. (2018) 
TABLE 5 | (Continued) Phytochemicals of Gynura species.

\begin{tabular}{|c|c|c|}
\hline Species & Identified phytochemicals & References \\
\hline Gynura divaricata (L.) DC. & $\begin{array}{l}\text { 3,4-Dicaffeoylquinic acid (Isochlorogenic acid B) (PubChem CID: } \\
\text { 5281780) } \\
\text { 3,5-Dicaffeoylquinic acid (Isochlorogenic acid A) (PubChem CID: } \\
\text { 6474310) } \\
\text { 4,5-Dicaffeoylquinic acid (Isochlorogenic acid C) (PubChem CID: } \\
\text { 6474309) } \\
\text { 3-Caffeoylquinic acid (Chlorogenic acid) (PubChem CID: } \\
\text { 1794427) } \\
\text { Cubenol (PubChem CID: 519857) } \\
\text { Spathulenol (PubChem CID: 92231) }\end{array}$ & Jiangseubchatveera et al. (2015), Dong et al. (2019) \\
\hline Gynura segetum (Lour.) Merr. & $\begin{array}{l}\text { Rutin (PubChem CID: 5280805) } \\
\text { Gallic acid (PubChem CID: 370) } \\
\text { 4,5,4'-Trihydroxychalcone (PubChem CID: 468135) } \\
\text { 8,8'-(Ethene-1,2-diyl)-dinaphtalene-1,4,5-triol (PubChem CID: } \\
\text { not found) }\end{array}$ & Yuandani et al. (2017) \\
\hline Gynura nepalensis DC. & $\begin{array}{l}\text { 3,4-Dicaffeoylquinic acid methyl ester (PubChem CID: not found) } \\
\text { 3,5-Dicaffeoylquinic acid ethyl ester (PubChem CID: not found) } \\
\text { 3,5-Dicaffeoylquinic acid methyl ester (PubChem CID: 10075681) } \\
\text { 3-O-cis-p-Coumaroylquinic acid (PubChem CID: 9945785) } \\
\text { 4,5-Dicaffeoylquinic acid methyl ester (PubChem CID: not found) } \\
\text { Chlorogenic acid (PubChem CID: 1794427) } \\
\text { Isochlorogenic acid A (PubChem CID: 6474310) } \\
\text { Isochlorogenic acid B (PubChem CID: 5281780) } \\
\text { Isochlorogenic acid C (PubChem CID: 6474309) } \\
\text { Saponins (PubChem CID: 6540709) } \\
\text { Tannins (PubChem CID: 250395) }\end{array}$ & Yu et al. (2016), Aktar et al. (2019) \\
\hline
\end{tabular}

to pathogenesis of various diseases. Thus the plants in genus Gynura are having high potential to be explored for pharmacological evidence of different ailments.

\section{FUTURE PERSPECTIVE}

Previous studies on Gynura species have indicated that several Gynura species possessed strong antioxidant and anti-inflammatory effects that are fundamental to various therapeutic purposes. Hence, Gynura species are potential continual source of new and useful bioactive compounds. Identification of the bioactive compounds in the plants contributing to the bioactivities, along with their mechanisms of action responsible for the pharmacological activities, is needed. Table 5 shows the list of phytochemicals identified in Gynura species. There is a need to investigate whether the crude extracts or isolated pure compounds are more effective. Synergistic effects of multiple active compounds in the extract may lead to a stronger pharmacological effect than that achievable by a single compound. At this stage, limited clinical studies on the antioxidant and anti-inflammatory effects of genus Gynura have been conducted. Human clinical trials with clearly defined symptomology to evaluate the therapeutic value of genus Gynura are necessary because animal experiments cannot be a substitute for clinical trials in the evaluation of therapeutic efficacy. To the best of our knowledge, there is only one non-systematic randomized controlled trial that has been carried out. Rerknimitr et al. (2016) investigated the efficacy of G. pseudochina DC. var. hispida Thv. ointment in treating chronic plaque psoriasis in a randomized controlled trial.

The bioavailability of bioactive molecules from genus Gynura also has limited investigation. On the basis of the study by $\mathrm{Wu}$ et al. (2015), the use of G. bicolor extract showed improvement on in vivo iron absorption and storage protein, which might be related to its rich phytoactive ingredients. Although the traditional uses of genus Gynura are supported by scientific evidence, the processing, and application or consumption methods by the public may alter the phytochemical profile, thus leading to pharmacological effect variations. Herbal combination is possible to provide better effects or benefits in developing therapeutic drugs. The study by Sari et al. (2015) showed that the combination of Andrographis paniculata (Burm. f.) Ness and G. procumbens could be a potential candidate for the development of an antidiabetic agent. The optimum therapeutic effect could be attributed to the combination of the potent hypoglycemic effect of $A$. paniculata and the potent antioxidant effect of G. procumbens. Hence, further studies on the therapeutic effects of the combinations of Gynura species with other medicinal plants are potentially producing more effective disease remedies. However, the potential issues of herb-herb and herb-drug interactions should be given due consideration, and further studies are needed to ensure no adverse interactions in polypharmacy and polyherbacy conditions. 


\section{CONCLUSION}

The extracts and phytochemicals of several Gynura species, particularly G. procumbens, G. bicolor, G segetum, G. divaricata, G. formosana, G. nepalensis, and G. pseudochina, have been reported to exhibit strong antioxidant and anti-inflammatory effects. However, in vitro and in vivo studies and clinical studies that have been carried out on the species are by no means free of methodological flaws. All 27 studies selected in this systematic review depicted risk of bias at different extent. The lack of randomization, lack of blinding, and unclear methodology explanation are some prevalent limitations. Further preclinical studies, including toxicity and pharmacokinetic studies on Gynura extracts and their bioactive compounds, are necessary before they can be subjected to clinical studies. Genus Gynura has high potential to be developed into medicinal agents for prophylactic supplements of diseases related to oxidative stress or inflammation.

\section{AUTHOR CONTRIBUTIONS}

TJN, ZJ, and NMF designed the study. IJ and KH provided information on the ethnopharmacology of Gynura and

\section{REFERENCES}

Akowuah, G. A., Ahmad, M., and Fei, M. Y., (2012). Effects of Gynura procumbens leaf extracts on plasma lipid peroxidation and total antioxidant status in $\mathrm{CCl}_{4}$ treated rats. Nat. Prod. J. 2 (4), 247-251. doi:10.2174/2210315511202040247

Akowuah, G. A., Mariam, A., and Chin, J. H. (2009). The effect of extraction temperature on total phenols and antioxidant activity of Gynura procumbens leaf. Phcog. Mag. 4 (17), 81-85.

Akowuah, G. A., Sadikun, A., and Mariam, A. (2002). Flavonoid identification and hypoglycaemic studies of the butanol fraction from Gynura procumbens. Pharmaceut. Biol. 40 (6), 405-410. doi:10.1076/phbi.40.6.405.8440

Aktar, A., Hafiz Hassan, S. M., Parvin, T., Akhlas, M. B., Khatun, F., Islam, M. T., et al. (2019). Further phytochemical screening; non-clinical evaluation of toxic and anti-inflammatory effects of crude aqueous extract of Gynura nepalensis. Pharmacologyonline 1, 136-153. Available at: https://pharmacologyonline.silae. it/files/archives/2019/vol1/PhOL_2019_1_A015_Anzuman.pdf

Algariri, K., Atangwho, I. J., Meng, K. Y., Asmawi, M. Z., Sadikun, A., and Murugaiyah, V. (2014). Antihyperglycaemic and toxicological evaluations of extract and fractions of Gynura procumbens leaves. Trop. Life Sci. Res. 25 (1), 75-93. Available at: https://www.ncbi.nlm.nih.gov/pmc/articles/PMC4156475/

Amelang, E., Prasad, C. M., Raymond, R. M., and Grega, G. J. (1981). Interactions among inflammatory mediators on edema formation in the canine forelimb. Circ. Res. 49 (2), 298-306. doi:10.1161/01.RES.49.2.298

Arulselvan, P., Fard, M. T., Tan, W. S., Gothai, S., Fakurazi, S., Norhaizan, M. E., et al. (2016). Role of antioxidants and natural products in inflammation. Oxid. Med. Cell Longev. 2016, 5276130. doi:10.1155/2016/5276130

Ayala, A., Muñoz, M. F., and Argüelles, S. (2014). Lipid peroxidation: production, metabolism and signaling mechanisms of malondialdehyde and 4-hydroxy-2nonenal. Oxid. Med. Cell. Longev. 2014, 360438. doi:10.1155/2014/360438

Biswas, S. K. (2016). Does the interdependence between oxidative stress and inflammation explain the antioxidant paradox? Oxid. Med. Cell. Longev. 2016, 1-9. doi:10.1155/2016/5698931

Chakravarthi, S., Jessop, C. E., and Bulleid, N. J. (2006). The role of glutathione in disulphide bond formation and endoplasmic-reticulum-generated oxidative stress. EMBO Rep. 7 (3), 271-275. doi:10.1038/sj.embor.7400645

Chandradevan, M., Simoh, S., Mediani, A., Ismail, I. S., and Abas, F. (2020). ${ }^{1}$ H NMRbased metabolomics approach in investigating the chemical profile, antioxidant sources of Gynura research. TJN conducted the literature search, extracted the data, and wrote the first draft. ZJ and NMF oversaw the research project, including checking the research work, reviewing, and interpreting the results. SMS and FB provided methodological advice on the project (literature search, screening, and selection). All authors are involved in reviewing and approval of the final manuscript.

\section{FUNDING}

We would like to thank the Ministry of Higher Education for FRGS grant FRGS/1/2019/SKK06/UKM/02/1.

\section{ACKNOWLEDGMENTS}

The authors would like to thank Ernieda Md Hatah for her advice on the issues raised during the review process. The authors would like to thank Enago (www.enago.com) for the English language review.

and anti-inflammatory activities of Gynura procumbens and Cleome gynandra. Plant Foods Hum. Nutr. 75, 243-251. doi:10.1007/s11130-020-00805-3

Chao, C., Liu, W., Wu, J., and Yin, M. (2015). Phytochemical profile, antioxidative and anti-inflammatory potentials of Gynura bicolor DC. J. Sci. Food Agric. 95 (5), 1088-1093. doi:10.1002/jsfa.6902

Chen, J., Lü, H., Fang, L. X., Li, W. L., Verschaeve, L., Wang, Z. T., et al. (2017). Detection and toxicity evaluation of pyrrolizidine alkaloids in medicinal plants Gynura bicolor and Gynura divaricata collected from different Chinese locations. Chem. Biodivers. 14 (2), e1600221. doi:10.1002/cbdv. 201600221

Chen, J., Mangelinckx, S., Lü, H., Wang, Z. T., Li, W. L., and De Kimpe, N. (2015). Profiling and elucidation of the phenolic compounds in the aerial parts of Gynura bicolor and $G$. divaricata collected from different Chinese origins. Chem. Biodivers. 12 (1), 96-115. doi:10.1002/cbdv.201400134

Chen, Z., Huo, J. R., Yang, L., and Zhu, H. Y. (2011). Effect of ligustrazine on mice model of hepatic veno-occlusive disease induced by Gynura segetum. J. Gastroenterol. Hepatol. 26 (6), 1016-1021. doi:10.1111/j.1440-1746.2011. 06661.x

Cuadrado, A., Kügler, S., and Lastres-Becker, I. (2018). Pharmacological targeting of GSK-3 and NRF2 provides neuroprotection in a preclinical model of tauopathy. Redox Biol. 14, 522-534. doi:10.1016/j.redox.2017.10.010

Davies, F. G. (1980). The genus Gynura (compositae) in India, Sri Lanka and the Seychelles. Kew Bull. 35 (2), 363. doi:10.2307/4114583

Dong, X., Zhao, S. X., Xu, B. Q., and Zhang, Y. Q. (2019). Gynura divaricata ameliorates hepatic insulin resistance by modulating insulin signalling, maintaining glycolipid homeostasis and reducing inflammation in type 2 diabetic mice. Toxicol. Res. 8 (6), 928-938. doi:10.1039/c9tx00191c

Ekor, M. (2014). The growing use of herbal medicines: issues relating to adverse reactions and challenges in monitoring safety. Front. Pharmacol. 4, 177. doi:10. 3389/fphar.2013.00177

Facco, M., Miorin, M., Agostini, C., and Semenzato, G. (2007). Granuloma formation. Progr. Respir. Res. 36, 87-100. doi:10.1159/000102629.

Ferlinahayati, F., Gultom, R. P. J., Herlina, H., and Eliza, E. (2017). Steroid compounds from Gynura pseudochina (lour) DC. Molekul 12 (1), 8-13. doi:10.20884/1.jm.2017.12.1.293

Folashade, O., Omoregie, H., and Ochogu, P. (2012). Standardization of herbal medicines-A review. Int. J. Biodivers. Conserv. 4 (3), 101-112. doi:10.5897/ IJBC11.163 
Forman, H. J., Zhang, H., and Rinna, A. (2009). Glutathione: overview of its protective roles, measurement, and biosynthesis. Mol. Aspect. Med. 30 (1-2), 1-12. doi:10.1016/j.mam.2008.08.006

Huang, X. L., Li, X. J., Qin, Q. F., Li, Y. S., Zhang, W. K., and Tang, H. B. (2019). Anti-inflammatory and antinociceptive effects of active ingredients in the essential oils from Gynura procumbens, a traditional medicine and a new and popular food material. J. Ethnopharmacol. 239, 111916. doi:10.1016/j.jep. 2019.111916

Ighodaro, O. M., and Akinloye, O. A. (2018). First line defence antioxidants-superoxide dismutase (SOD), catalase (CAT) and glutathione peroxidase $(\mathrm{GPX})$ : their fundamental role in the entire antioxidant defence grid. Alexandria J. Med. 54 (4), 287-293. doi:10. 1016/j.ajme.2017.09.001

Iskander, M. N., Song, Y., Coupar, I. M., and Jiratchariyakul, W. (2002). Antiinflammatory screening of the medicinal plant Gynura procumbens. Plant Foods Hum. Nutr. 57 (3-4), 233-244. doi:10.1023/a: 1021851230890

Jiangseubchatveera, N., Liawruangrath, B., Liawruangrath, S., Korth, J., and Pyne, S. G. (2015). The chemical constituents and biological activities of the essential oil and the extracts from leaves of Gynura divaricata (L.) DC. Growing in Thailand. J. Essent. Bearing Plants 18 (3), 543-555. doi:10.1080/0972060x.2014. 935016

Kaewseejan, N., and Siriamornpun, S. (2015). Bioactive components and properties of ethanolic extract and its fractions from Gynura procumbens leaves. Ind. Crop. Prod. 74, 271-278. doi:10.1016/j.indcrop.2015.05.019

Kaminska, B. (2005). MAPK signalling pathways as molecular targets for antiinflammatory therapy - from molecular mechanisms to therapeutic benefits. Biochim. Biophys. Acta 1754, 253-262. doi:10.1016/j.bbapap.2005.08.017

Kim, J., Lee, C. W., Kim, E. K., Lee, S. J., Park, N. H., Kim, H. S., et al. (2011). Inhibition effect of Gynura procumbens extract on UV-B-induced matrixmetalloproteinase expression in human dermal fibroblasts. J. Ethnopharmacol. 137 (1), 427-433. doi:10.1016/j.jep.2011.04.072

Kim, S. P., Ha, J. M., Yun, S. J., Kim, E. K., Chung, S. W., Hong, K. W., et al. (2010). Transcriptional activation of peroxisome proliferator-activated receptor- $\gamma$ requires activation of both protein kinase A and Akt during adipocyte differentiation. Biochem. Biophys. Res. Commun. 399 (1), 55-59. doi:10. 1016/j.bbrc.2010.07.038

Knekt, P., Järvinen, R., Reunanen, A., and Maatela, J. (1996). Flavonoid intake and coronary mortality in Finland: a cohort study. Br. Med. J. 312, 478. doi:10.1136/ bmj.312.7029.478

Krishnan, V., Ahmad, S., and Mahmood, M. (2015). Antioxidant potential in different parts and callus of Gynura procumbens and different parts of Gynura bicolor. BioMed Res. Int. 2015, 147909. doi:10.1155/2015/147909

Kumari, R., and Kotecha, M. (2016). A review on the standardization of herbal medicines. Int. J. Pharm. Sci. Res 7 (2), 97-106.

Legler, D. F., Bruckner, M., Uetz-von Allmen, E., and Krause, P. (2010). Prostaglandin E2 at new glance: novel insights in functional diversity offer therapeutic chances. Int. J. Biochem. Cell Biol. 42 (2), 198-201. doi:10.1016/j. biocel.2009.09.015

Li, D. P., Chen, Y. L., Jiang, H. Y., Chen, Y., Zeng, X. Q., Xu, L. L., et al. (2019). Phosphocreatine attenuates Gynura segetum-induced hepatocyte apoptosis via a SIRT3-SOD2-mitochondrial reactive oxygen species pathway. Drug Des. Dev. Ther. 13, 2081-2096. doi:doi:10.2147/DDDT.S203564

Li, J., Qin, Y., Yu, X., Xiong, Z., Zheng, L., Sun, Y., et al. (2018). In vitro simulated digestion and in vivo metabolism of chlorogenic acid dimer from Gynura procumbens (Lour.) Merr.: enhanced antioxidant activity and different metabolites of blood and urine. J. Food Biochem. 43, e12654. doi:10.1111/jfbc.12654

Li, S., Han, Q., Qiao, C., Song, J., Cheng, C. L., and Xu, H. (2008). Chemical markers for the quality control of herbal medicines: an overview. Chin. Med. 3, 7. doi:10. 1186/1749-8546-3-7

Liu, M., He, M., Gao, H., Guo, S., Jia, J., Ouyang, H., et al. (2019). Strategy for rapid screening of antioxidant and anti-inflammatory active ingredients in Gynura procumbens (Lour.) Merr. based on UHPLC-Q-TOF-MS/MS and characteristic ion filtration. Biomed. Chromatogr. 33, e4635. doi:10.1002/bmc.4635

Liu, T., Zhang, L., Joo, D., and Sun, S. C. (2017). NF-kB signaling in inflammation. Signal Transduc. Target. Ther. 2 (1), 1-9. doi:10.1038/sigtrans.2017.23

Liu, Y., You, J., Xu, W., Zhai, T., Du, C., Chen, Y., et al. (2019). Gynura procumbens aqueous extract alleviates nonalcoholic steatohepatitis through CFLAR-JNK pathway in vivo and in vitro. Chin. Herb. Med. 11 (4), 369-378. doi:10.1016/j. chmed.2019.09.005

Liu, Z., Ren, Z., Zhang, J., Chuang, C. C., Kandaswamy, E., Zhou, T., et al. (2018). Role of ROS and nutritional antioxidants in human diseases. Front. Physiol. 9, 477. doi:10.3389/fphys.2018.00477

Lu, C.-H., Yang, H.-C., Chang, W.-L., Chang, Y.-P., Wu, C.-C., and Hsieh, S.-L. (2012). Development of beverage product from Gynura bicolor and evaluation of its antioxidant activity. Genomic Med., Biomarkers, Health Sci. 4 (4), 131-135. doi:10.1016/j.gmbhs.2013.04.001

Ma, J., Guo, C., Pan, Y., Lin, D., Qiu, L., and Wen, L. (2017). Antioxidant and antiinflammatory activities of ethyl acetate extract of Gynura formosana (Kitam) leaves. Exper. Ther. Med. 14 (3), 2303-2309. doi:10.3892/etm.2017.4757

Manandhar, N. P. (2002). Plants and people of Nepal. Plants and people of Nepal. Timber Press: Portland.

Manicone, A. M., and McGuire, J. K. (2008). Matrix metalloproteinases as modulators of inflammation. Semin. Cell Dev. Biol. 19 (1), 34-41. doi:10. 1016/j.semcdb.2007.07.003

Mittal, M., Siddiqui, M. R., Tran, K., Reddy, S. P., and Malik, A. B. (2014). Reactive oxygen species in inflammation and tissue injury. Antioxid. Redox Signal 20 (7), 1126-1167. doi:10.1089/ars.2012.5149

Moher, D., Liberati, A., Tetzlaff, J., and Altman, D. G. (2010). Preferred reporting items for systematic reviews and meta-analyses: the PRISMA statement. Int. J. Surg. 8 (5), 336-341. doi:10.1016/J.IJSU.2010.02.007

Muraina, I., Adaudi, A., Mamman, M., Kazeem, H., and Eloff, J. (2008). Effects of geographical location on the yield and bioactivity of Anoigeissus leiocarpus. J. Pharm. Bioresour. 5 (2), 68-72. doi:10.4314/jpb.v5i2.52995

Murugaiyah, V., Ahmed Saeed, M., Kuong, Y.-M., Murugesu, K., Parasuraman, S., Asmawi, M. Z., et al. (2018). Lipid-lowering effect of hydroalcoholic extracts of Gynura procumbens in chemical- and High-fat diet-induced hyperlipidemic rats. Phcog. Mag. 14 (55), 184-191. doi:10.4103/pm. pm_451_17

Murugesu, K., Murugaiyah, V., Saghir, S. A. M., Asmawi, M. Z., and Sadikun, A. (2017). Caffeoylquinic acids rich versus poor fractions of Gynura procumbens: their comparative antihyperlipidemic and antioxidant potential. Curr. Pharmaceut. Biotechnol. 18 (14), 1132-1140. doi:10.2174/1389201019666180322111800

Nazri, K. A. A., Fauzi, N. M., Buang, F., Mohd Saad, Q. H., Husain, K., Jantan, I., et al. (2019). Gynura procumbens standardised extract reduces cholesterol levels and modulates oxidative status in postmenopausal rats fed with cholesterol diet enriched with repeatedly heated palm oil. Evid. Base Compl. Alternat. Med. 2019, 1-15. doi:10.1155/2019/7246756

Ning, T. J., Yusoff, S. D., Jubri, Z., Buang, F., Song, T. Z., Budiono, A., et al. (2019). Inhibitory effects of Gynura procumbens ethanolic extract on nitric oxide production and inducible nitric oxide synthase (iNOS) protein expression in macrophages. Sains Malays. 48 (8), 1737-1744. doi:10. 17576/jsm-2019-4808-20

Pai, P., Mong, M., Yang, Y., Liu, Y., Wang, Z., and Yin, M. (2019). Anti-diabetic effects of Gynura bicolor aqueous extract in mice. J. Food Sci. 84 (6), 1631-1637. doi:10.1111/1750-3841.14607

Qiu, S., Zhang, H., Fei, Q., Zhu, F., Wang, J., Jia, X., et al. (2018). Urine and plasma metabolomics study on potential hepatoxic biomarkers identification in rats induced by Gynura segetum. J. Ethnopharmacol. 216, 37-46. doi:10.1016/j.jep. 2018.01.017

Qiu, X. L., Guo, Y. X., and Zhang, Q. F. (2018). Chemical profile and antioxidant activity of Gynura bicolor dc. Ethanolic extract. Int. J. Food Prop. 21 (1), 407-415. doi:10.1080/10942912.2018.1424199

Rahman, M. M., Rahman, M. H., Oliullah, Md. A. B., and Shahriar, M. (2018). Antiinflammatory and analgesic activities of ethanolic extract of Gynura nepalensis (leaf). Pharmacologyonline 3, 247-253. Available at: https://pharmacologyonline. silae.it/files/archives/2018/vol3/PhOL_2018_3_A027_Rahman.pdf

Rerknimitr, P., Nitinawarat, J., Weschawalit, S., Wititsuwannakul, J., Wongtrakul, P., Jutiviboonsuk, A., et al. (2016). The Efficacy of Gynura pseudochina DC. var. hispida Thv. Ointment in treating chronic plaque psoriasis: a randomized controlled trial. J. Alternative Compl. Med. 22 (8), 669-675. doi:10.1089/acm. 2016.0100

Rivera, D., Allkin, R., Obón, C., Alcaraz, F., Verpoorte, R., and Heinrich, M. (2014). What is in a name? the need for accurate scientific nomenclature for plants. J. Ethnopharmacol. 152 (3), 393-402. doi:10.1016/j.jep.2013. 12.022 
Rooney, A. A., Boyles, A. L., Wolfe, M. S., Bucher, J. R., and Thayer, K. A. (2014). Systematic review and evidence integration for literature-based environmental health science assessments. Environ. Health Perspect. 122 (7), 711-718. doi:10. 1289/ehp.1307972

RosidahMun, F. Y., Sadikun, A., Ahmad, M., Akowuah, G. A., and Asmawi, M. Z. (2009). Toxicology evaluation of standardized methanol extract of Gynura procumbens. J. Ethnopharmacol. 123, 244-249. doi:10.1016/j.jep. 2009.03.011

RosidahYam, M., Sadikun, A., and Asmawi, M. (2008). Antioxidant potential of Gynura procumbens. Pharmaceut. Biol. 46 (9), 616-625. doi:10.1080/ 13880200802179642

Salazar, M., Rojo, A. I., Velasco, D., De Sagarra, R. M., and Cuadrado, A. (2006). Glycogen synthase kinase- $3 \beta$ inhibits the xenobiotic and antioxidant cell response by direct phosphorylation and nuclear exclusion of the transcription factor Nrf2. J. Biol. Chem. 281 (21), 14841-14851. doi:10.1074/ jbc.M513737200

Samuel, A. J. S. J., Kalusalingam, A., Chellappan, D. K., Gopinath, R., Radhamani, S., Husain, H. A., et al. (2010). Ethnomedical survey of plants used by the Orang Asli in Kampung Bawong, Perak, west Malaysia. J. Ethnobiol. Ethnomed. 6 (1), 1-6. doi:10.1186/1746-4269-6-5

Sari, K. R. P., Sudarsonoand Nugroho, A. E. (2015). Effect of herbal combination of Andrographis paniculata (Burm.f) Ness and Gynura procumbens (Lour.) Merr ethanolic extracts in alloxan-induced hyperglycemic rats. Int. Food Res. J. 22 (4), 1332-1337. Available at: http://www.ifrj.upm.edu.my/22\%20(04)\%202015/(3). pdf

Seow, L. J., Beh, H. K., Umar, M. I., Sadikun, A., and Asmawi, M. Z. (2014). Antiinflammatory and antioxidant activities of the methanol extract of Gynura segetum leaf. Int. Immunopharm. 23 (1), 186-191. doi:10.1016/j.intimp.2014. 08.020

Sharma, J. N., Al-Omran, A., and Parvathy, S. S. (2007). Role of nitric oxide in inflammatory diseases. Inflammopharmacology 15 (6), 252-259. doi:10.1007/ s10787-007-0013-x

Shwter, A. N., Abdullah, N. A., Alshawsh, M. A., Alsalahi, A., Hajrezaei, M., Almaqrami, A. A., et al. (2014). Chemoprevention of colonic aberrant crypt foci by Gynura procumbens in rats. J. Ethnopharmacol. 151 (3), 1194-1201. doi:10. 1016/j.jep.2013.12.044

Sies, H. (1993). Strategies of antioxidant defense. Eur. J. Biochem. 215 (2), 213-219. doi:10.1111/j.1432-1033.1993.tb18025.x

Siriwatanametanon, N., Fiebich, B. L., Efferth, T., Prieto, J. M., and Heinrich, M. (2010). Traditionally used Thai medicinal plants: in vitro anti-inflammatory, anticancer and antioxidant activities. J. Ethnopharmacol. 130 (2), 196-207. doi:10.1016/j.jep.2010.04.036

Siriwatanametanon, N., and Heinrich, M. (2011). The Thai medicinal plant Gynura pseudochina var. hispida: chemical composition and in vitro NF-kappaB inhibitory activity. Nat. Prod. Commun. 6 (5), 627-630. doi:10.1177/ $1934578 \times 1100600512$

Stocker, R., McDonagh, A. F., Glazer, A. N., and Ames, B. N. (1990). Antioxidant activities of bile pigments: biliverdin and bilirubin. Methods Enzymol. 186 (C), 301-309. doi:10.1016/0076-6879(90)86123-D

Sukadeetad, K., Nakbanpote, W., Heinrich, M., and Nuengchamnong, N. (2018). Effect of drying methods and solvent extraction on the phenolic compounds of Gynura pseudochina (L.) DC. leaf extracts and their anti-psoriatic property. Ind. Crop. Prod. 120, 34-46. doi:10.1016/j.indcrop.2018.04.020

Teoh, W. Y., Sim, K. S., Moses Richardson, J. S., Abdul Wahab, N., and Hoe, S. Z. (2013). Antioxidant capacity, cytotoxicity, and acute oral toxicity of Gynura bicolor. Evid. Base Compl. Alternat. Med. 2013, 958407. doi:10.1155/2013/ 958407

Teoh, W. Y., Wahab, N. A., Richardson, J. S. M., and Sim, K. S. (2016). Evaluation of antioxidant properties, cytotoxicity and acute oral toxicity of Gynura procumbens (Compositae). Sains Malays. 45 (2), 229-235. http://www.ukm. my/jsm/pdf_files/SM-PDF-45-2-2016/10\%20Wuen\%20Yew\%20Teoh.pdf

The Plant List (2020). Version 1.1. Available at: http://www.theplantlist.org/ (Accessed January 21, 2020).

Townsend, D. M., and Tew, K. D. (2003). The role of glutathione-S-transferase in anti-cancer drug resistance. Oncogene 22 (47), 7369-7375. doi:10.1038/sj.onc. 1206940

Turkseven, S., Kruger, A., Mingone, C. J., Kaminski, P., Inaba, M., Rodella, L. F., et al. (2005). Antioxidant mechanism of heme oxygenase-1 involves an increase in superoxide dismutase and catalase in experimental diabetes. Am. J. Physiol. Heart Circ. Physiol. 289, H701-H707. doi:10.1152/ajpheart.00024.2005

Turner, M. D., Nedjai, B., Hurst, T., and Pennington, D. J. (2014). Cytokines and chemokines: at the crossroads of cell signalling and inflammatory disease. Biochim. Biophys. Acta Mol. Cell Res. 1843 (11), 2563-2582. doi:10.1016/j. bbamcr.2014.05.014

Tyagi, S., Gupta, P., Saini, A., Kaushal, C., and Sharma, S. (2011). The peroxisome proliferator-activated receptor: a family of nuclear receptors role in various diseases. J. Adv. Pharm. Technol. Res. 2 (4), 236-240. doi:10.4103/2231-4040. 90879

Upton, R., David, B., Gafner, S., and Glasl, S. (2019). Botanical ingredientidentification and quality assessment: strengths and limitations of analytical techniques. Phytochem. Rev. 19, 1-21. doi:10.1007/s11101-019-09625-z

Valavanidis, A., Vlachogianni, T., and Fiotakis, C. (2009). 8-Hydroxy-2' -deoxyguanosine (8-OHdG): a critical biomarker of oxidative stress and carcinogenesis. J. Environ. Sci. Health C Environ. Carcinog. Ecotoxicol. Rev. 27 (2), 120-139. doi:10.1080/10590500902885684

Vanhaesebroeck, B., and Alessi, D. R. (2000). The PI3K-PDK1 connection : more than just a road to PKB. Biochem. J. 346, 561-576. doi:10.1042/bj3460561

Vanijajiva, O., and Kadereit, J. W. (2011). A revision of Gynura (Asteraceae: Senecioneae). J. Systemat. Evol. 49 (4), 285-314. doi:10.1111/j.1759-6831.2011. 00139.x

Vaziri, N. D., and Rodríguez-Iturbe, B. (2006). Mechanisms of disease: oxidative stress and inflammation in the pathogenesis of hypertension. Nat. Clin. Pract. Nephrol. 2 (10), 582-593. doi:10.1038/ncpneph0283

Vejanan, V., Latip, J., Lee, P. C., Embi, N., and Sidek, H. M. (2012). In vitro and in vivo anti-plasmodial activities of Gynura procumbens. Sains Malays. 41 (12), $1535-1542$.

Wachtel-Galor, S., and Benzie, I. F. F. (2011). "Herbal medicine: an introduction to its history, usage, regulation, current trends, and research needs." in Herbal medicine: biomolecular and clinical aspects. 2nd Edn. Boca Raton, Florida: CRC Press, 1-10.

Wan, C., Yu, Y., Zhou, S., Liu, W., Tian, S., and Cao, S. (2011). Antioxidant activity and free radical-scavenging capac ity of Gynura divaricata leaf extracts at different temperatures. Phcog. Mag. 7 (25), 40. doi:10.4103/0973-1296.75900

Wang, H., Kumar, A., Lamont, R. J., and Scott, D. A. (2014). GSK3 $\beta$ and the control of infectious bacterial diseases. Trends Microbiol. 22 (4), 208-217. doi:10.1016/j. tim.2014.01.009

Wiart, C. (2006). Medicinal plants of the aisa-pacific: drugs for the future? Singapore, Singapore: World ScientificWorld Scientific Publishing Co.

Williams, G. T., and Williams, J. (1983). Granulomatous inflammation-a review. J. Clin. Pathol. 36, 723-733. doi:10.1136/jcp.36.7.723

Wong, S. K., Jann, M. L. S., Sudi, S., Hassan, W. R. B. M., Chin, L. P., Embi, N., et al. (2015). Anti-malarial and anti-inflammatory effects of Gynura procumbens are mediated by kaempferol via inhibition of glycogen

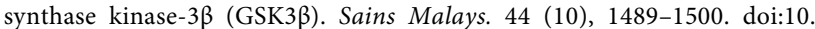
17576/jsm-2015-4410-15

Wu, C. C., Chang, W. L., Lu, C. H., Chang, Y. P., Wang, J. J., and Hsieh, S. L. (2015). Effects of extracts from Gynura bicolor (Roxb. \& Willd.) DC. on iron bioavailability in rats. J. Food Drug Anal. 23 (3), 425-432. doi:10.1016/j.jfda. 2014.11.001

Wu, C. C., Lii, C. K., Liu, K. L., Chen, P. Y., and Hsieh, S. L. (2013). Antiinflammatory activity of Gynura bicolor (紅鳳菜 Hóng Fèng Cài) ether extract through inhibits Nuclear Factor Kappa B activation. J. Tradit. Comple. Med. 3 (1), 48-52. doi:10.1016/s2225-4110(16)30166-3

Xiong, A., Shao, Y., Fang, L., Xiao, Y., Zhang, S., Zheng, J., et al. (2018). Comparative analysis of toxic components in different medicinal parts of Gynura japonica and its toxicity assessment on mice. Phytomedicine 54, 77-88. doi:10.1016/j.phymed.2018.06.015

Xu, B. Q., Yang, P., and Zhang, Y. Q. (2015). Hypoglycemic activities of lyophilized powder of Gynura divaricata by improving antioxidant potential and insulin signaling in type 2 diabetic mice. Food Nutr. Res. 59, 29652. doi:10.3402/fnr.v59. 29652

Yang, Y. C., Wu, W. T., Mong, M. C., and Wang, Z. H. (2019). Gynura bicolor aqueous extract attenuated $\mathrm{H} 2 \mathrm{O} 2$ induced injury in PC12 cells. Biomedicine 9 (2), 38-45. doi:10.1051/bmdcn/2019090212

Yin, M., Wang, Z., Liu, W., and Mong, M. (2017). Aqueous extract of Gynura bicolor attenuated hepatic steatosis, glycative, oxidative, and inflammatory 
injury induced by chronic ethanol consumption in mice. J. Food Sci. 82 (11), 2746-2751. doi:10.1111/1750-3841.13930

Yu, B. W., Li, J. L., Guo, B. B., Fan, H. M., Zhao, W. M., and Wang, H. Y. (2016). Chlorogenic acid analogues from Gynura nepalensis protect $\mathrm{H} 9 \mathrm{c} 2$ cardiomyoblasts against $\mathrm{H} 2 \mathrm{O} 2$-induced apoptosis. Acta Pharmacolo. Sin. 37 (11), 1413-1422. doi:10.1038/aps.2016.79

YuandaniJantan, I., and Husain, K. (2017). 4,5,4'-Trihydroxychalcone, 8,8'(ethene-1,2-diyl)-dinaphtalene-1,4,5-triol and rutin from Gynura segetum inhibit phagocytosis, lymphocyte proliferation, cytokine release and nitric oxide production from phagocytic cells. BMC Complement. Alternat. Med. 17 (1), 211. doi:10.1186/s12906-017-1726-Z

Yue, L., and Yao, H. (2016). Mitochondrial dysfunction in inflammatory responses and cellular senescence: pathogenesis and pharmacological targets for chronic lung diseases. Br. J. Pharmacol. 173 (15), 2305-2318. doi:10.1111/bph.13518

Zahra, A. A., Kadir, F. A., Mahmood, A. A., Al hadi, A. A., Suzy, S. M., Sabri, S. Z., et al. (2011). Acute toxicity study and wound healing potential of Gynura procumbens leaf extract in rats. J. Med. Plants Res. 5 (12), 2551-2558.

Zhang, F., Zhou, Y., Yang, X., Xiong, A., Wang, Z., and Yang, L. (2019). Gynura Rhizoma containing pyrrolizidine alkaloids induces the hepatic sinusoidal obstruction syndrome in mice via upregulating fibrosis-related factors. Acta Pharmacol. Sin. 40 (6), 781-789. doi:10.1038/s41401-018-0155-y
Zhang, J. M., and An, J. (2007). Cytokines, inflammation, and pain. Int. Anesthesiol. Clin. 45 (2), 27-37. doi:10.1097/AIA.0b013e318034194e

Zhu, H., Chu, Y., Huo, J., Chen, Z., and Yang, L. (2011). Effect of prednisone on transforming growth factor- $\beta 1$, connective tissue growth factor, nuclear factor$\kappa B p 65$ and tumor necrosis factor- $\alpha$ expression in a murine model of hepatic sinusoidal obstruction syndrome induced by Gynura segetum. Hepatol. Res. 41 (8), 795-803. doi:10.1111/j.1872-034X.2011.00830.x

Zumla, A., and James, D. G. (1996). Granulomatous infections: etiology and classification. Clin. Infect. Dis. 23, 146-158. doi:10.1093/clinids/23.1.146

Conflict of Interest: The authors declare that the research was conducted in the absence of any commercial or financial relationships that could be construed as a potential conflict of interest.

Copyright (C) 2020 Tan, Mohd Saffian, Buang, Jubri, Jantan, Husain and Mohd Fauzi. This is an open-access article distributed under the terms of the Creative Commons Attribution License (CC BY). The use, distribution or reproduction in other forums is permitted, provided the original author(s) and the copyright owner(s) are credited and that the original publication in this journal is cited, in accordance with accepted academic practice. No use, distribution or reproduction is permitted which does not comply with these terms. 